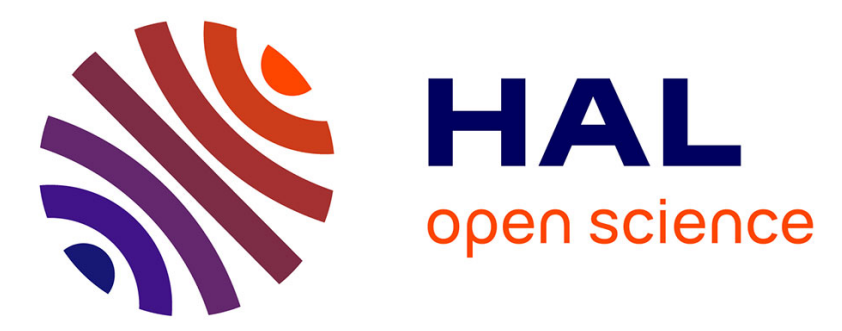

\title{
Long-gage optical fiber extensometers for dynamic evaluation of structures
}

\author{
Gwendal Cumunel, S. Delepine-Lesoille, Pierre Argoul
}

\section{To cite this version:}

Gwendal Cumunel, S. Delepine-Lesoille, Pierre Argoul. Long-gage optical fiber extensometers for dynamic evaluation of structures. Sensors and Actuators A: Physical , 2012, 184, pp.1-15. 10.1016/j.sna.2012.06.005 . hal-00829935

\section{HAL Id: hal-00829935 \\ https: / hal-enpc.archives-ouvertes.fr/hal-00829935}

Submitted on 5 Jun 2013

HAL is a multi-disciplinary open access archive for the deposit and dissemination of scientific research documents, whether they are published or not. The documents may come from teaching and research institutions in France or abroad, or from public or private research centers.
L'archive ouverte pluridisciplinaire HAL, est destinée au dépôt et à la diffusion de documents scientifiques de niveau recherche, publiés ou non, émanant des établissements d'enseignement et de recherche français ou étrangers, des laboratoires publics ou privés. 


\title{
Long-gage optical fiber extensometers for dynamic evaluation of structures
}

\author{
G.Cumunel $^{\mathrm{a}, 1, *}$, S.Delepine-Lesoille $\mathrm{a}^{\mathrm{a}, 2}$, P.Argoul $^{\mathrm{b}}$ \\ ${ }^{a}$ Université Paris-Est, IFSTTAR, 58, boulevard Lefebvre, 75732 Paris Cedex 15, France \\ ${ }^{b}$ Université Paris-Est, Laboratoire NAVIER (ENPC/IFSTTAR/CNRS), Ecole des Ponts ParisTech, 6 et 8 Avenue \\ Blaise Pascal, 77455 Marne-la-Vallée, France
}

\begin{abstract}
This paper deals with the study of the capacities of continuously attached long-gage fiber optic sensors for a new use: dynamic evaluation of structures.

The optical system is first presented, followed by the development of the precise formulation of the measurement data obtained by this sensor when applied to the dynamic analysis of beams, especially under bending oscillations. This sensor allows to reach the curvature mode shapes and numerical simulations are then performed to estimate the dynamic characteristics of the beam by means of the continuous wavelet transform, from the data obtained with this sensor.

Finally, the fiber optic sensors are bonded on a real cantilever beam and experimental data are achieved from the optical measurement system, in the case of after shock free oscillations of the instrumented beam. A similar modal identification procedure as that proposed for numerical simulations is used and the results are compared to those obtained with accelerometers and long strain gauges. This type of sensor allowing to get the curvature mode shapes will be a good candidate for damage detection.
\end{abstract}

\section{Keywords: long-gage extensometer / fiber optic sensor / structural dynamics / modal analysis / Euler-Bernoulli beam / wavelet transform}

\section{Introduction}

Experimental dynamic evaluation of mechanical structures is a thriving field of research. Recent literature mainly deals with sophisticated methods of experimental data processing [1]. However, the choices regarding the type of sensor and its location remain determinant factors. The use of accelerometers is largely predominant, although their size is sometimes too small compared to the size of the structure. Moreover, total cost of instrumentation is especially significant because many sensors must be installed if the global behavior is to be measured instead of local effects. For that reason, the use of fewer sensors is often preferred, e.g., the multi-patch technique. Some sensors are fixed so as to serve as references, while others are moved along the entire structure [2]. When a

\footnotetext{
${ }^{*}$ Corresponding author

Email addresses: gwendal.cumunel@enpc.fr (G.Cumunel), Sylvie.Lesoille@andra.fr (S.Delepine-Lesoille), pierre.argoul@enpc.fr (P.Argoul)

${ }^{1}$ Present address: Université Paris-Est, Laboratoire NAVIER (ENPC/IFSTTAR/CNRS), Ecole des Ponts ParisTech, 6 et 8 Avenue Blaise Pascal, 77455 Marne-la-Vallée, France

${ }^{2}$ Present address: ANDRA, Parc de la Croix Blanche 1/7 rue Jean Monnet 92298 Chatenay-Malabry France
} 
small number of sensors is chosen, deciding their respective locations becomes a rather painstaking process $[3,4]$. A possible alternative to this is the use of Long-Gage Fiber Optic Sensors (LGFOS) $[5,6]$.

Several types of optical fiber extensometers exist [7], among which Bragg gratings [7, 8] are the most popular. In fact, this kind of Fiber Optic Sensor (FOS), which is a few centimeters long, has been the only reliable interferometric sensor enabling dynamic measurements for the last decade $[9,10]$. As a result, apart from their multiplexing capabilities, electromagnetic neutrality, and robustness, they are used exactly in the same way as standard extensometers. This article focuses on another type of optical extensometer providing very long gage lengths of up to several meters that still conserve resolution at the micrometer level. This specification is of major importance if a real decametric structure has to be instrumented for a reasonable price.

Long-gage fiber optic extensometers have been commercially available for more than a decade [5] and are mounted on many structures for long-term monitoring [11, 12], especially on civil engineering structures $[13,14]$. They are made of two optical fiber portions whose lengths are originally strictly identical. One dummy arm is isolated from the structure while the other measuring arm is attached to it. As the structure exhibits strains, either stretch or compression, the fiber bonded to, or embedded into, the structure elongates or shortens accordingly. Based on low-coherence interferometry, the interrogation unit is able to determine the length difference between the two optical fibers. Because optical fiber is a waveguide, measurement corresponds to the integral of the curvilinear abscissa along the measuring optical fiber, and not to the Euclidean distance provided by a vibrating wire sensor. The first commercially-available sensor sheath was a tube containing both fibers, with one left loose inside the tube and the other tightened between two anchoring points [5]. A few years ago, other types of sensor sheaths were developed that preserve the flexibility of the optical fiber and allow them to be attached continuously to structures $[15,16]$. In the following, CA-LGFOS stands for Continuously-Attached Long-Gage Fiber Optic Sensor. Their major applications were for static monitoring, but recently they have been used for dynamic studies. Very few dynamic experiments have been reported in literature $[14,17,18]$ and are limited to potential experimental applications. This article focuses on the use of CA-LGFOS for the dynamic evaluation of structures. Our work concerns the evaluation of the capacity of this type of sensor when applied for dynamics purposes.

The paper is organized as follows: Section 2 describes the measurement principle of the optical device and the sensor that was used for the laboratory dynamic experiments presented in Section 5. In Section 3, the formulation of the measurement performed by such sensors is elaborated upon and applied to a linear dynamic analysis of an Euler-Bernoulli beam for bending vibrations. A numerical modal analysis is achieved in Section 4 through an application of the Continuous Wavelet Transform (CWT) method so as to determine the dynamic characteristics obtained from the sensor. Finally, experimental validation on a plexiglass cantilever beam with accelerometers, strain gauges, and CA-LGFOS is presented and discussed in Section 5.

\section{Optical measurement system}

The FOS (Fig.1(a)) consists of a single-mode optical fiber comprising of a Fabry-Perot cavity with two semi-reflective mirrors in the fiber. The mirrors are semi-reflective for serial multiplexing. These mirrors (Fig.1(b)) can, for example, be produced by the end-to-end assembling of two optical fibers treated beforehand with fine layers of dielectric so as to choose the desired mirror reflectivities. 
[Figure 1 about here.]

The measurement system prototype is based on a low-coherence interferometer, similar to the system proposed in [19], adapted to provide dynamics measurements. The measurement arm is continuously attached to the structure, and the reference arm is deported into the measurement unit [6]. The system measures the change in path distance between partial mirrors formed on a single-mode optical fiber of any arbitrary length, and it will be referred to as the "sensor" (Fig. 1). The reading unit (Fig. 2) incorporates a photodetector, reference arm, measuring arm with a mobile mirror (scanning a few millimeters long path), and a diode (SLED at $1310 \mathrm{~nm}$ ). The reference and measuring arms constitute the delay line of the system. Half of the diode light illuminates the sensor, which reflects a portion of this incident light into the delay line. The delay line analyses the reflected light, and signals are recombined on the photodetector. When the time delay between the two reflections at both ends of the sensor is reproduced into the delay line, an interference peak a few microns wide (the coherence length of the source) occurs [20,21]. Knowing the position of the mirror, the length of the cavity (or sensor) can be measured. Any changes in the sensor's length can be detected by a shift of the signal peak to a different location detected by the reading unit. The total displacement over the gage length is then measured. It is assumed that thermal effects are corrected by the allocation of a dummy gauge or other compensation schemes.

[Figure 2 about here.]

Although low-coherence interferometry has been deployed with great success for more than a decade with the well-known SOFO system [5], the proposed scheme is innovative since it takes advantage of two specificities. First, the translation stage of the system is made of a very compact and stable electromagnet where the mirror is attached, as will be reported in great details in a future publication. It enables dynamic measurements with a sampling frequency of $100 \mathrm{~Hz}$ (fixed) and a measurement range of $4 \mathrm{~mm}$ (between $\pm 2 \mathrm{~mm}$ ). Second, since the reference arm is located inside the measurement unit, various sensors can be multiplexed in series (as long as their length fits in the measuring range). Moreover, this configuration provides a great flexibility due to the sensor coating. There is no need for an intrusive splitter at the location of the first mirror of the sensor (where a reference arm is traditionally located). Thus, the sensor coating may remain as flexible as the pristine optical fiber, so as to be continuously attached to the structure to monitor. A drawback is temperature compensation. Since the sensor and the reference arm are not co-located, they do not undergo the same temperature. This is why it has been proposed and implemented to use the same low-coherence interferometer to also monitor fiber Bragg gratings dedicated to structure temperature sensing, as detailed in [19]. For sake of simplicity and as it is out of scope of the proposed dynamic evaluation, this feature will not be mentioned in the following.

\section{Theoretical measurement performed by the CA-LGFOS}

To determine the measurement obtained by this type of optical reading unit, which is equal to the phase shift produced by strain undergone by the FOS, many authors [20, 22, 23, 24] use Eqs. (1) and (2):

$$
\begin{gathered}
L_{o p t}=n L \\
\Delta x=n \Delta L+L \Delta n
\end{gathered}
$$


with $n$ the refractive index of the optical fiber, $L$ the physical length of the sensor, $\Delta x$ the mirror displacement, and $\Delta L$ and $\Delta n$ the variation of the physical length and of the refractive index due to strain, respectively.

However, as evidenced in [25], it is not always clearly mentioned that these equations are only valid for a straight FOS undergoing a linear or a constant strain field. In this paper, the formulation of the measurement performed by CA-LGFOS is developed, without simplifications, for a sensor of any shape bonded to, or embedded into a solid submitted to a linear strain field.

As previously mentioned, CA-LGFOS allows measurements between two particular points along the optical fiber, which are determined by reflectivities enclosed inside the silica waveguide. More precisely, the theoretical temporal measurement performed by CA-LGFOS is given by Fermat's principle [21]:

$$
L_{o p t}\left(s_{a}, s_{b}, t\right)=\int_{s_{a}}^{s_{b}} n(t) d s(t)
$$

where $s_{a}$ and $s_{b}$ are the abscissas of the flexible sensor extremities (in $m$ ), $s$ indicating the curvilinear abscissa. This last parameter is defined as the ratio between the speed of light in the material and in vacuum. It depends on temperature and strain, as thoroughly evidenced in [23, 25, 26]. The measurement obtained by the optical reading unit is then:

$$
\Delta x=L_{o p t}\left(s_{a}, s_{b}, t\right)-L_{o p t}\left(s_{a}, s_{b}, 0\right)
$$

To obtain $L_{o p t}\left(s_{a}, s_{b}, t\right)$ given in Eq. (3), the curvilinear abscissa variation $d s$ and the refractive index $n$ have to be determined. Thus, this section is dedicated to the expression of these terms and to their dependence on temperature, strain, and optical fiber sheath. Only the main relations will be indicated. More details are given in Appendix.

\subsection{Determination of the curvilinear abscissa variation and refractive index}

The expression of $d s$ is classically defined using the components $\varepsilon_{i j}$ of the Green-Lagrange strain tensor:

$$
d s^{2}=d s_{0}^{2}+2 \varepsilon_{i j} d x_{i} d x_{j} \quad i, j=1, \ldots, 3
$$

where

$$
\left\{\begin{aligned}
\varepsilon_{i j} & =\frac{1}{2}\left(\frac{\partial U_{i}}{\partial x_{j}}+\frac{\partial U_{j}}{\partial x_{i}}+\frac{\partial U_{k}}{\partial x_{i}} \cdot \frac{\partial U_{k}}{\partial x_{j}}\right) \quad k=1, \ldots, 3 \\
d s_{0}^{2} & =g_{i j} d x_{i} d x_{j} \\
g_{i j} & =\frac{\partial \overrightarrow{O M}}{\partial x_{i}} \cdot \frac{\partial \overrightarrow{O M}}{\partial x_{j}}
\end{aligned}\right.
$$

where $d s_{0}$ is the initial curvilinear abscissa, $g_{i j}$ the components of the metric tensor, $\overrightarrow{O M}$ the vector defining the initial position of the CA-LGFOS located inside or on the structure and $U=\left[U_{1}, U_{2}, U_{3}\right]$ the displacement field vector of the structure in the Euclidean reference $O x_{1} x_{2} x_{3}$.

The refractive index is estimated by a constant $n_{0}$ at a first order approximation. It differs according to the propagation medium of the electromagnetic wave; for example, $n_{0}=1.3$ and 1.5 for water and glass, respectively. However, the refractive index varies due to the effect of external actions. Parameters generally taken into account for the computation of the index variation are temperature and strain. The dependence of the refractive index on strain is called the photoelastic effect and is usually taken into account $[25,26,27,28,29,30]$. The refractive index dependence 
on temperature is experimentally determined [31].

After calculation (see Appendix) for an optical fiber along $x_{1}$-axis, the refractive index can be expressed by:

$$
n=\frac{n_{0}}{2}\left(\frac{1}{\left.\sqrt{1+n_{0}^{2} p_{2 k} \beta_{k}\left(\varepsilon_{k}+\alpha_{k}^{\text {int }} \Delta T\right.}\right)}+\frac{1}{\sqrt{1+n_{0}^{2} p_{3 k} \beta_{k}\left(\varepsilon_{k}+\alpha_{k}^{i n t} \Delta T\right)}}\right)+\frac{\partial n}{\partial T} \Delta T \quad k=1, \ldots, 6
$$

where $n_{0}$ is the constant part of the refractive index, $\beta_{k}$ are the components of a tensor introduced for strain transfer, $p_{2 k}$ and $p_{3 k}$ are the photoelastic tensor components (in $B \mathrm{w}$ ), $\alpha_{k}^{\text {int }}$ are the components of a tensor taking into account the interactions between the incorporated optical fiber and the thermal expansions of the host material (in $K^{-1}$ or ${ }^{\circ} C^{-1}$ ), $\frac{\partial n}{\partial T}$ is the refractive index dependent on temperature (in $K^{-1}$ or ${ }^{\circ} C^{-1}$ ), and $\Delta T$ is the temperature variation (in $K$ or ${ }^{\circ} C$ ). The contracted notation used for a symmetric tensor "S" is defined by:

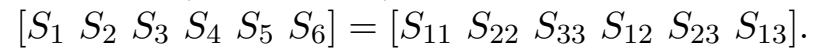

So, by gathering the influence of the various parameters (strain, temperature, and coating), the measurement of CA-LGFOS becomes:

$L_{\text {opt }}\left(s_{a}, s_{b}, t\right)=\int_{s_{a}}^{s_{b}}\left[\frac{n_{0}}{2}\left(\frac{1}{\sqrt{1+n_{0}^{2} p_{2 k} \beta_{k}\left(\varepsilon_{k}+\alpha_{k}^{\text {int }} \Delta T\right)}}+\frac{1}{\sqrt{1+n_{0}^{2} p_{3 k} \beta_{k}\left(\varepsilon_{k}+\alpha_{k}^{\text {int }} \Delta T\right)}}\right)+\frac{\partial n}{\partial T} \Delta T\right] d s$

where $d s=\sqrt{\left[g_{k}+2 \beta_{k}\left(\varepsilon_{k}+\alpha_{k}^{i n t} \Delta T\right)\right] d x_{k}}$ and $k=1, \ldots, 6$.

In the following, temperature influence will be neglected. We neglect it first, because dynamic experiments are often quite fast compared to temperature fluctuations, and, secondly, because the optoelectronic interrogation unit usually takes this phenomenon into account. This is why the model would strongly depend on the measurement system's architecture (association with either dummy gauge $[15,20]$ or punctual temperature Bragg grating [19, 29]). Then, Eq. (8) is restricted to:

$$
L_{o p t}\left(s_{a}, s_{b}, t\right)=\int_{s_{a}}^{s_{b}} \frac{n_{0}}{2}\left(\frac{1}{\sqrt{1+n_{0}^{2} p_{2 k} \beta_{k} \varepsilon_{k}}}+\frac{1}{\sqrt{1+n_{0}^{2} p_{3 k} \beta_{k} \varepsilon_{k}}}\right) d s
$$

where $d s=\sqrt{\left[g_{k}+2 \beta_{k} \varepsilon_{k}\right] d x_{k}}$ and $k=1, \ldots, 6$.

\subsection{Application case: straight Euler-Bernoulli beam}

The application example, chosen for a detailed study of the CA-LGFOS measurement, is related to the bending vibrations of a slender beam in the plan $\left(O, x_{1}, x_{2}\right)$ (Fig. 3). Euler-Bernoulli kinematics assumptions are applied: the cross-section of the beam remains flat and perpendicular to the neutral axis during the strain of the beam. The initial beam configuration is such that the neutral axis orientation is along the $x_{1}$-axis and that the CA-LGFOS is located at a constant distance $x_{2}$ from the neutral axis.

[Figure 3 about here.] 
As $x_{2}$ is constant, the only dependent variable is $x_{1}$. The vector defining the position of the CA-LGFOS is $\overrightarrow{O M}=\left[\begin{array}{ll}x_{1} x_{2} & 0\end{array}\right]$, so $d s_{0}$ is equal to $\sqrt{g_{11} d x_{1}^{2}}=d x_{1}$. Consequently,

$$
d s=\sqrt{1+2 \beta_{11} \varepsilon_{11}} d x_{1} .
$$

When the optical fiber is along the $x_{1}$-axis, the average refractive index in each point of the optical fiber, defined in [6] as $n=\left(n_{x_{2}}+n_{x_{3}}\right) / 2$, is:

$$
n=\frac{n_{0}}{\sqrt{1+n_{0}^{2} \beta_{11}\left[(1-\nu) p_{12}-\nu p_{11}\right] \varepsilon_{11}}} .
$$

In conclusion, with Eqs. (10) and (11), the CA-LGFOS measurement can be determined to be:

$$
L_{\text {opt }}(a, b, t)=\int_{a}^{b} \frac{n_{0}}{\sqrt{1+n_{0}^{2} \beta_{11}\left[(1-\nu) p_{12}-\nu p_{11}\right] \varepsilon_{11}(t)}} \cdot \sqrt{1+2 \beta_{11} \varepsilon_{11}(t)} d x_{1}
$$

with $\varepsilon_{11}(t)$ the axial strain undergone by the fiber, $n_{0}=1.46$ the optical fiber refractive index (silica), $\nu=0.25$ the Poisson coefficient of the optical fiber, and $p_{11}=0.12$ and $p_{12}=0.27$ the optical fiber photoelastic constants (in $B \mathrm{w}$ ) [22].

\subsection{Simplifying assumptions}

Usually small-strain assumption is carried out $\left(\varepsilon_{11}<<1\right)$ in order to simplify expressions. For the curvilinear abscissa variation $d s$ and the refractive index $n$, it leads to:

$$
\begin{aligned}
d s & \approx\left(1+\beta_{11} \varepsilon_{11}\right) d x_{1} \\
n & \approx n_{0}-\frac{1}{2} n_{0}^{3} \beta_{11}\left[(1-\nu) p_{12}-\nu p_{11}\right] \varepsilon_{11}=n_{0}+\kappa \cdot \varepsilon_{11}
\end{aligned}
$$

where $\kappa$ is a constant approximately equal to -0.27 when $\beta_{11}=1$.

CA-LGFOS measurement will then be approximated by:

$$
\Delta L_{o p t}(a, b, t) \approx\left(\beta_{11} n_{0}+\kappa\right) \int_{a}^{b} \varepsilon_{11}(t) d x_{1}
$$

with $\Delta L_{o p t}(a, b, t)=L_{o p t}(a, b, t)-L_{o p t}(a, b, 0)$ and $L_{o p t}(a, b, 0)=n_{0} \int_{a}^{b} d x_{1}=n_{0}(b-a)$.

The physical length of the sensor $L_{p h y s}(a, b, t)$ is defined by

$$
L_{\text {phys }}(a, b, t)=\int_{s_{a}}^{s_{b}} d s=\int_{a}^{b} \sqrt{1+2 \beta_{11} \varepsilon_{11}(t)} d x_{1} .
$$

With small-strain assumption, the optical length variation $\Delta L_{o p t}(a, b, t)$ is proportional to the physical length variation $\Delta L_{\text {phys }}(a, b, t)$ :

$$
\Delta L_{\text {opt }}(a, b, t)=\frac{\left(\beta_{11} n_{0}+\kappa\right)}{\beta_{11}} \cdot \Delta L_{p h y s}(a, b, t) .
$$

Thus, we will now be only interested in the calculation of the physical length $L_{p h y s}(a, b, t)$ and its variation $\Delta L_{\text {phys }}(a, b, t)$. Moreover, we associate $\Delta L_{\text {phys }}(a, b, t)$ with the CA-LGFOS measurement. It should also be noted that the optical length variation measurement is about $19 \%$ greater than the length variation undergone by the optical fiber when $\beta_{11}=1$. 


\subsection{CA-LGFOS aftershock oscillations}

Linear beam aftershock vibrations in the $\left(O, x_{1}, x_{2}\right)$ plane, calculated with Euler-Bernoulli kinematics assumptions, are studied. The beam's transverse displacement response $\left(U_{2}=v\left(x_{1}, t\right)\right)$ to a shock along $x_{2}$-axis, when restricted to the $N$ first modes, is classically given by modal superposition [32]:

$$
v\left(x_{1}, t\right) \approx \sum_{k=1}^{N} b_{k}(t) \phi_{k}\left(x_{1}\right)
$$

where $b_{k}(t)$ and $\phi_{k}\left(x_{1}\right)$ are respectively the $k^{t h}$ modal response to the force $g\left(x_{1}, t\right)$ and the $k^{t h}$ beam mode shape.

The impact force of amplitude $F$ is applied to the beam at time $t=t_{0}$ and abscissa $x_{1}=x_{0}$, and is defined by:

$$
g\left(x_{1}, t\right)=F \cdot \delta^{t_{0}}(t) \cdot \delta^{x_{0}}(x)
$$

where $\delta^{t_{0}}(t)$ and $\delta^{x_{0}}(x)$ are Dirac distribution in time and in space, respectively.

The response $b_{k}(t)$ to the force $g\left(x_{1}, t\right)$ is then defined by the Duhamel's integral:

$$
b_{k}(t)=\frac{1}{\rho S \tilde{\omega}_{k}} \int_{0}^{t} F_{k}(\tau) e^{-\xi_{k} \omega_{k}(t-\tau)} \cdot \sin \left(\tilde{\omega}_{k}(t-\tau)\right) d \tau
$$

where $F_{k}(\tau)=\int_{0}^{L} g\left(x_{1}, \tau\right) \phi_{k}\left(x_{1}\right) d x_{1}, \quad \tilde{\omega}_{k}=\omega_{k} \sqrt{1-\xi_{k}^{2}}$, and $\omega_{k}=\frac{\left(\gamma_{k} L\right)^{2}}{L^{2}} \sqrt{\frac{E_{0} I}{\rho S}} . E_{0}$ is the Young modulus of the beam (in $G P a$ ), $S$ the beam cross section (in $m^{2}$ ), $\rho$ the beam mass density (in $\mathrm{kg} \cdot \mathrm{m}^{-3}$ ), $L$ the beam length (in $\mathrm{m}$ ), I the area moment of inertia of the beam section (in $m^{4}$ ), $\tilde{\omega}_{k}$ the reduced pulsation (in rad.s ${ }^{-1}$ ), $\omega_{k}$ the pulsation (in rad.s ${ }^{-1}$ ), and $\xi_{k}$ the damping ratio of the $k^{t h}$ mode of the beam. $\gamma_{k} L$ is a solution of the transcendental equation corresponding to boundary conditions studied; for example $\cos \left(\gamma_{k} L\right) \cdot \cosh \left(\gamma_{k} L\right)=-1$ for clamped-free conditions.

After calculations, it leads to:

$$
b_{k}(t)=\frac{F \phi_{k}\left(x_{0}\right)}{\rho S \tilde{\omega}_{k}} \sin \left(\tilde{\omega}_{k}\left(t-t_{0}\right)\right) \cdot e^{-\xi_{k} \omega_{k}\left(t-t_{0}\right)} .
$$

The expression of the mode shapes depends on the boundary conditions. For example, for clamped-free conditions we have:

$$
\phi_{k}=\frac{a_{k}}{\sqrt{\rho S L}}\left[\frac{\cos \left(\beta_{k} L\right)+\cosh \left(\beta_{k} L\right)}{\sinh \left(\beta_{k} L\right)+\sin \left(\beta_{k} L\right)}\left(\sin \left(\beta_{k} x_{1}\right)-\sinh \left(\beta_{k} x_{1}\right)\right)+\cosh \left(\beta_{k} x_{1}\right)-\cos \left(\beta_{k} x_{1}\right)\right] .
$$

where $a_{k}$ is a factor introduced to scale the mode shapes to unit modal masses.

Modal analysis induces the linearity and stationarity of the system's behavior. In this case, the strain is simply approximated by $\varepsilon_{11} \approx-x_{2} v^{\prime \prime}\left(x_{1}, t\right)$, where $x_{2}$ is the distance of the sensor from the beam's neutral axis, that is assumed to be constant, and $v^{\prime \prime}\left(x_{1}, t\right)$ is the second spatial derivative of the beam displacement, which is equal to curvature when the dynamic behavior of the beam is linear. CA-LGFOS measurement is then:

$$
\Delta L_{\text {phys }}(a, b, t)=-x_{2} \int_{a}^{b} v^{\prime \prime}\left(x_{1}, t\right) d x_{1} .
$$


Using Eq. (17) leads to:

$$
\Delta L_{p h y s}(a, b, t) \approx-x_{2} \sum_{k=1}^{N} b_{k}(t) \Phi_{k}(a, b)
$$

where

$$
\Phi_{k}(a, b)=\int_{a}^{b} \phi_{k}^{\prime \prime}\left(x_{1}\right) d x_{1}=\phi_{k}^{\prime}(b)-\phi_{k}^{\prime}(a)
$$

with $\phi_{k}^{\prime \prime}\left(x_{1}\right)$ representing the $k^{t h}$ modal curvature of the beam and $\phi_{k}^{\prime}\left(x_{1}\right)$ the "modal rotations."

Eq. (23) shows the modal parameters that can be obtained with CA-LGFOS. As eigenfrequencies and modal damping ratios are obtained with the temporal factor $b_{k}(t)$, we can say that CA-LGFOS measurement is similar to a displacement. This result is not surprising as CA-LGFOS measures a length variation. However, the spatial term $\Phi_{k}(a, b)$ is different from classical punctual sensors (displacement sensor, velocimeter, or accelerometer) firstly because it depends on two points, the mirror locations $a$ and $b$, instead of one, the sensor location $x_{1}^{i}$. Secondly, $\phi_{k}\left(x_{1}^{i}\right)$ are modal displacements, whereas $\Phi_{k}(a, b)$ are modal curvature integrals between the mirrors of the sensor, or in other words, modal rotation differences at the points where mirrors are located. They will be called curvature mode shapes.

The average curvature is used in statics [33]. Eq. (23), which directly connects CA-LGFOS measurement to modal curvature, motivated several research teams to work on damage detection methods based on modal curvature [17, 34].

\section{Numerical modal analysis: CA-LGFOS and standard punctual sensors}

A numerical modal analysis is performed on a clamped-clamped beam case. The beam is homogeneous with a constant rectangular section $S=h \cdot e$ and length $L$. The excitation force is modeled by an impact $g\left(x_{1}, t\right)$ applied to $x_{1}$-coordinate $x_{0}=0.95 \mathrm{~m}$ at time $t_{0}=0$. Beam characteristics for numerical study are indexed in Table 1. Simulated measurements of the different sensor types are obtained: displacement, velocity and acceleration (from Eq. (17) and its derivatives) and CA-LGFOS (from Eq. (23)). Fast Fourier Transforms (FFT) are then calculated. Measurements are performed between $t_{0}$ and $t_{f}$, which is a time interval discretized in $N_{t}$ points. Parameter values chosen for numerical study are indexed in Table 2 . We will only be interested in the first three modes of the clamped-clamped beam, and the number of sensors is equal to ten for each type of measurement. Sensor locations are indexed in Table 3.

[Table 1 about here.]

[Table 2 about here.]

[Table 3 about here.]

The modal analysis method used is an output-only method based on the Continuous Wavelet Transform (CWT) applied to aftershock responses [35, 36]. In [36], Argoul and Le illustrated with several examples that the use of the CWT has many advantages for the processing of transient 
signals in civil engineering because of its simplicity of implementation, quality of obtained information, and better comprehensibility of the studied phenomenon, especially when nonlinear effects occur. As this type of method is output-only, normalization to the maximum is applied for observed mode shapes. The mother wavelets used for signal processing are Cauchy wavelets. The method uses specific parameters: stop time $t_{f}$ of the processed signals, a quality factor $Q$, the frequency bandwidth being studied, and the number of points discretizing this frequency interval $N_{c w t}$. For more details on this method, refer to [35]. Fig. 4 presents the global CWT of the first sensor for the different types of measurement on the frequency bandwidth [0-40] $\mathrm{Hz}$. The stop time is equal to the total duration of the signal, so $t_{f}=15 \mathrm{~s}, Q=20$, and $N_{c w t}=400$ points. Similar graphs are obtained for other sensors. Then local treatment of the signals in the time-frequency plan is carried out, and these parameters are indexed in Table 4.

[Figure 4 about here.]

[Table 4 about here.]

As expected, the CA-LGFOS measurement is similar to a displacement measurement for the energy repartition on the CWT graph, so these sensors are more sensitive to low frequencies.

Eigenfrequencies and damping ratios determined for the first three modes by the CWT method correspond extremely well to the theoretical ones. Observed mode shapes obtained with the CWT method also correspond extremely well to the theoretical ones determined from punctual sensors and CA-LGFOS. Thus, we confirm that curvature mode shapes are obtained with CA-LGFOS. In Fig. 5, the observed numerical mode shapes are depicted for punctual sensors and CA-LGFOS. For CA-LGFOS graphs, the theoretical curve plotted for each mode is the modal curvature $\phi_{k}^{\prime \prime}\left(x_{1}\right)$.

[Figure 5 about here.]

Fig. 5 shows the particular mode shapes obtained from CA-LGFOS measurement. Indeed, as their values are the integral of the modal curvatures, each one is not associated with a point but with the measurement basis of CA-LGFOS. So the curvature mode shapes will be steps curves, or a bars diagram [37]. However, for a continuous distribution of CA-LGFOS along the beam, the "modal rotations" discretized at the CA-LGFOS mirrors locations, $\phi_{k}^{\prime}\left(x_{i}\right)$, can be obtained by adding the curvature mode shapes values of the sensors as defined in Eq. (25):

$$
\begin{array}{ccc}
\sum_{j=1}^{1}\left[\phi_{k}^{\prime}\left(x_{1}^{j}\right)-\phi_{k}^{\prime}\left(x_{1}^{j-1}\right)\right] & = & \phi_{k}^{\prime}\left(x_{1}^{1}\right)-\phi_{k}^{\prime}\left(x_{1}^{0}\right) \\
\vdots & \vdots & \vdots \\
\sum_{j=1}^{i}\left[\phi_{k}^{\prime}\left(x_{1}^{j}\right)-\phi_{k}^{\prime}\left(x_{1}^{j-1}\right)\right] & = & \phi_{k}^{\prime}\left(x_{1}^{i}\right)-\phi_{k}^{\prime}\left(x_{1}^{0}\right) \\
\vdots & \vdots & \vdots \\
\sum_{j=1}^{n}\left[\phi_{k}^{\prime}\left(x_{1}^{j}\right)-\phi_{k}^{\prime}\left(x_{1}^{j-1}\right)\right] & & \phi_{k}^{\prime}\left(x_{1}^{n}\right)-\phi_{k}^{\prime}\left(x_{1}^{0}\right)
\end{array}
$$

The constant $\phi_{k}^{\prime}\left(x_{1}^{0}\right)$ is zero for the clamped-clamped beam. Then, by interpolating and integrating the discretized "modal rotations", the mode shapes $\phi_{k}\left(x_{1}\right)$ can be determined. In practice, 
this is not recommended because the dispersions of the experimental results are amplified by the numerical interpolation and integration.

Discretized modal curvatures $\phi_{k}^{\prime \prime}\left(x_{1}\right)$ can also be obtained with punctual sensors by numerical second order differentiation [38], using central finite difference scheme. To limit the amplification of experimental dispersions due to numerical differentiation, high-order digital filtering is applied. So, the information of numerous points at each end of the sensors distribution are lost.

\section{Experimental modal analysis with CA-LGFOS}

This section is devoted to a modal analysis performed on a cantilever beam in order to test the capacities of CA-LGFOS for dynamic structural analysis. First, it is important to note some limitations of the optical measurement system.

The measurement unit was designed for dynamic monitoring of civil engineering structures, which have low frequency vibrational modes. Typically, the frequencies of the first vibration modes are of a few $\mathrm{Hz}$. Main specification was also to enable multiplexing. The characteristics of the optical measurement system finally obtained are a sampling frequency of $100 \mathrm{~Hz}$, a measurement range of $4 \mathrm{~mm}$ between $\pm 2 \mathrm{~mm}$ and an accuracy of about $0.7 \mu \mathrm{m}$ regardless the sensor length. The experimental validations of potential performances of the system were performed on laboratory experiments as beam-like structure. As the frequency bandwidth observable by the measurement unit is low (up to $50 \mathrm{~Hz}$ ) and several modes are to be observed in the CA-LGFOS responses, experiments design is a trade-off. To lower eigenfrequencies of the beam, the choice of the beam material is plexiglass, the boundary conditions are clamped-free, and the beam has to be quite long and thin. However if the thickness of the beam is too thin, strain is not sufficient to obtain a correct signal/noise ratio of CA-LGFOS measurement. What is more, the dynamic behavior of the beam is subjected to geometrical nonlinearities ${ }^{1}$ because of the boundary conditions and the beam's thickness.

Another limitation is the accuracy of the CA-LGFOS length. The manufacturing process of CA-LGFOS is not automated and the accuracy of the length of the sensor is of the order of one millimeter. Thus, on all the $20 \mathrm{~cm}$-long CA-LGFOS at our disposal, the maximum number of sensors that can be detected together by the measurement unit is three.

The plexiglass cantilever beam characteristics are indexed in Table 5 . The beam is instrumented with eight accelerometers, four $12 \mathrm{~cm}$-long strain gauges (usually used for concrete), and three 20 $\mathrm{cm}$-long CA-LGFOS (FOGALE nanotech brand). This beam is excited by an impact hammer. The excitation location is near the beam's free end. Since the strain gauges are continuously attached to the beam surface, as are CA-LGFOS, we assume as a first approximation that the sensors measure the physical length variation $\Delta L_{\text {phys }}(a, b, t)$. With the refractive index difference, the gauges will validate the CA-LGFOS measurement. With these dimensions of the beam, the strain amplitude is not very high, and, therefore, the Signal-to-Noise Ratio (SNR) of CA-LGFOS measurement signals will be too. As the excitation is an impact, the SNR definition used is $\mathrm{SNR}=20 \cdot \log _{10}\left(A_{\max } / A_{\text {noise }}\right)$, where $A_{\max }$ is the maximum of the absolute value of the signal and $A_{\text {noise }}$ the noise amplitude. For this experiment, the SNR of the measurement signals is between $26 d B$ and $32 d B$ for the CA-LGFOS, $44 d B$ and $54 d B$ for the strain gauges and $65 d B$ and $90 d B$ for the accelerometers.

\footnotetext{
${ }^{1}$ Moderate or large rotations of beam sections whose level is related to that of the excitation.
} 
The sensor locations are indexed in Table 6 and illustrated in Fig. 6. A photography of the beam with the various sensors is presented in Fig. 7. Accelerometers and gauges are attached on the same face of the beam and CA-LGFOS on the opposite face.

[Table 5 about here.]

[Figure 6 about here.]

[Table 6 about here.]

[Figure 7 about here.]

Accelerometer and gauge signals are directly conditioned and digitized by the Spider8 HBM system. Moreover, signals are synchronized with the impact hammer signal (pre-trigger), and the frequency sampling is equal to $1.2 \mathrm{kHz}$. CA-LGFOS measurements are obtained by the optical measuring device from FOGALE nanotech. The optical measuring device cannot be synchronized with other signals, so its measurement begins slightly before the impact was made. The frequency sampling of the optical measuring device FOGALE is technologically fixed and limited to $100 \mathrm{~Hz}$. Fig. 8 presents the temporal signals and FFT obtained with accelerometer $n^{\circ} 8$, strain gauge $n^{\circ} 1$, and CA-LGFOS $n^{\circ} 1$ on the cantilever beam that was excited by a $3 N$ impact applied at $x_{0}=0.98$ $m$.

[Figure 8 about here.]

Tests with low amplitude impacts were carried out in order to minimize the geometrical nonlinearities effect. Sensor response signals were then processed with the classic peak picking and the CWT methods in order to obtain modal parameters. Only the first two modes of the beam are studied. Tables 7 and 8 present the mean values and dispersions, respectively, of the eigenfrequencies and modal damping ratios, and of the observed mode shapes of the two first modes that were obtained with the accelerometers, strain gauges, and CA-LGFOS. Fig. 9 illustrates the observed mode shapes. In Fig. 9(c), 9(d), 9(e), and 9(f), the dotted curves represent the modal curvature $\phi_{1}^{\prime \prime}\left(x_{1}\right)$ and $\phi_{2}^{\prime \prime}\left(x_{1}\right)$ of the cantilever beam, respectively.

[Table 7 about here.]

[Figure 9 about here.]

[Table 8 about here.]

In Table 8, the mode shapes determined with the two methods are similar. For the eigenfrequencies and modal damping ratios, Table 7 shows that the two modal identification techniques give rather different results, especially for the first mode. Moreover, with the classic peak picking method, eigenfrequency and modal damping ratio values of the first mode differ among the sensor types and even among the same type sensors. The reason of these differences is that the behavior of the beam is not linear. Indeed, the CWT method can access to both instantaneous frequencies and damping ratios that have to be constant in time when the structure behavior is linear. The instantaneous frequencies of the first two modes obtained by CWT method with measurement signals of the accelerometers, strain gauges and CA-LGFOS are plotted in Fig. 10 and illustrate 
the nonlinear behavior of the beam. The black lines are parabolas representing the limits of validity range of the CWT, i.e., the range not submitted to edge effects (for details, see [35]). The trends of the instantaneous frequencies are comparable for the different sensor types. However, the curves are more fluctuant when the SNR of the mode component in the signal is low (the three CA-LGFOS for the second mode and one for the first mode) and rather different when the sensor is near a node of the mode ${ }^{1}$ (CA-LGFOS $n^{\circ} 3$ and accelerometer $n^{\circ} 1$ for the first mode). A "softening" effect ${ }^{2}$ due to geometrical nonlinearities is observed for the two modes, especially for the first one (variation of $\approx 10 \%$ in the validity range of the CWT against only $\approx 0.5 \%$ for the second mode, which can be considered almost linear).

As the behavior of the beam is not linear, the CWT values presented in Table 7 are the average of the instantaneous frequencies and damping ratios in the CWT validity range. This choice is arbitrary but allows to compare the different sensor types more precisely than with the classic peak picking method. Indeed in this nonlinear context, the CWT method is much more adapted and reliable for modal identification than the classic peak picking method. So, in the following, we will only use the results of the CWT method to compare the different sensor types.

[Figure 10 about here.]

The results of experimental modal identification do not correspond well with a finite element model even with the mass of the accelerometers taken into account: only the second frequency is quite well updated (error of $2.2 \%$ ); the error on the first frequency is about $25 \%$; the mode shapes are also quite different ( $\phi_{1}$ and $\phi_{2}$ in Table 8$)$ and the same goes for the curvature mode shapes ( $\Phi_{1}$ and $\Phi_{2}$ in Table 8). It's probably due to the nonlinear behavior of the cantilever beam and to the effect of the accelerometer cables (the damping ratio induced for the first mode is very high). The updating of the finite element model using experimental modal data is then difficult and is not the purpose of the experimental study. So, to appreciate the performances of the CA-LGFOS measurement, we will look at the dispersion values of the modal parameters and the comparison of the eigenfrequencies and modal damping ratios determined from the other sensor types.

First, it should be noted that the different estimates of eigenfrequency and modal damping ratio as well as the high values of dispersion obtained by accelerometer $n^{\circ} 1$, strain gauge $n^{\circ} 4$ and CA-LGFOS $n^{\circ} 3$ in Tables 7 and 8 are due to the sensors location close to a node of the mode shape. The eigenfrequencies of the first mode for the different sensor types in Table 7 are very similar $(0.01 \mathrm{~Hz}$ difference for average of the CA-LGFOS). However the dispersion values are a little higher for CA-LGFOS $(0.5 \%$ more). The estimates of the modal damping ratio are more difficult to analyse because, generally, the determination of this parameter is not very accurate. Moreover, as the behavior of the beam is not linear, the dispersion values for all the sensors are much more important than usual, especially for the first mode. For an unknown reason, it seems that these values are comparable for the extensometric sensor type $(\approx 7.4 \%)$ and differ from those obtained by the accelerometers $(\approx 6.4 \%)$. Such as for the eigenfrequencies, the dispersion values of the modal damping ratios are a little higher for CA-LGFOS, principally due to the low SNR of the sensors for this experiment.

The eigenfrequencies and modal damping ratios of the second mode in Table 7 are quasi identical for each type of sensors because the mode is almost linear. Only the dispersion values for the modal

\footnotetext{
${ }^{1}$ Close to the clamp for the accelerometers and close to the free end for the strain gauges and CA-LGFOS.

${ }^{2}$ The instantaneous frequency increases with time.
} 
damping ratio are more important for the CA-LGFOS. In Table 8, such as for the eigenfrequencies and modal damping ratios, the dispersion values of CA-LGFOS are higher than those obtained by the accelerometers and the strain gauges. The important one for the CA-LGFOS $n^{\circ} 3$ can be due to the impact applied in front of a mirror of this sensor, which is a sensitive part of the sensor, and also because this sensor has the lowest SNR.

The results obtained from CA-LGFOS for dynamic structural analysis of the beam prove that the technology of the optical measurement system and its sensor is not yet fully mature but the prospects are rather encouraging. To improve the dynamic structural analysis performances of the sensor, several points of the measurement system can be addressed such as increasing the sampling frequency or improving the manufacturing process of the sensor, for example. Among these, the increase of the sampling frequency is more important and can be easily achievable with more recent electromagnets or other recent translation stages but this could be quite expensive. Moreover, it could be done at the expense of multiplexing capabilities of the system, which depend of the measurement range. So, a compromise have to be found depending on the intended application between the number of sensors to use and the number of modes to observe. The accuracy of the system is also an important limitating aspect for laboratory experiments but it is limited by the wavelength of the light source and can not be drastically reduced because of the measuring principle of the optical system. However, if the sampling frequency is increased, thicker structures could be used for experiments and the SNR could be higher.

\section{Concluding remarks}

In this paper, we have proposed a complete formulation of the measurement obtained by Continuously Attached Fiber Optic Sensor and applied it to the dynamic analysis of beams under bending oscillations. The integral of modal curvatures of the beam are accessed by modal analysis of the CA-LGFOS measurement signals.

The optical measurement system used in the experiment, based on low coherence interferometry, has been presented as well as the fiber optic sensor. The measurement principle of the optical device is not new but has been adapted to provide dynamics measurements. The characteristics of the optical measurement unit are a sampling frequency of $100 \mathrm{~Hz}$ (fixed), a measurement range of 4 $m m$ between $\pm 2 \mathrm{~mm}$ and an accuracy of about $0.7 \mu \mathrm{m}$ regardless the sensor length.

Numerical and experimental modal analyses with CA-LGFOS have been performed on beamlike structures and the results have been compared to those obtained with other sensor types as accelerometers and long strain gauges. For the experimental study, the eigenfrequencies and modal damping ratios achieved through the Cauchy wavelet transform method with CA-LGFOS measurement signals, for the first two modes of a cantilever beam in the case of after shock free oscillations, are comparable to those obtained with accelerometers and strain gauges. However due to the low signal-to-noise ratio of CA-LGFOS measurement for this experiment, which is limited by the optical measurement system accuracy, the dispersion values for the determination of modal parameters is higher. To increase CA-LGFOS capabilities for vibration monitoring, some points of the system measurement can be improved such as the sampling frequency and the manufacturing process of the sensor. The assessment of CA-LGFOS for dynamic structural analysis of the beam is rather encouraging but the system needs some improvements to be even more reliable.

As curvature mode shapes can be directly obtained by CA-LGFOS; the major application of this sensor will be damage detection by modal curvature methods, e.g., the modal curvature, flexibility curvature, and strain energy methods. The use of this type of sensor would be more appropriate 
than accelerometers (or any other type of punctual sensor leading to modal displacements), which require numerical polynomial filtering and second order differentiation to access modal curvature.

\section{Acknowledgements}

The authors would like to thank Tiffany Hwang of Princeton University for her help in editing this article.

\section{Appendix A. Calculation of the measurement performed by CA-LGFOS}

This appendix presents some details on the formulation of the measurement performed by CA-LGFOS.

\section{Appendix A.1. Photoelastic effect}

The refractive index is estimated by a constant $n_{0}$ at a first order approximation. It differs according to the propagation medium of the electromagnetic wave; for example, $n_{0}=1.3$ and 1.5 for water and glass, respectively. However, the refractive index varies under the effect of external actions. Parameters generally taken into account for the computation of the index variation are temperature and strain. The dependence of the refractive index on strain is called the photoelastic effect. The principle will be briefly explained; for more details see [39].

The dielectric tensor is defined by:

$$
D=\epsilon E=\epsilon_{0} E+P
$$

with $D$ the dielectric tensor (in $C \cdot m^{-2}$ ), $E$ the electrical field vector (in $V \cdot m^{-1}$ ), $P$ the electrical polarization vector (in $C . m^{-2}$ ), $\epsilon_{0}$ the vacuum permittivity (in $F . m^{-1}$ ), and $\epsilon$ the dielectric tensor dependent on the medium (in F.m ${ }^{-1}$ ).

Contracted notations are used for additional simplicity:

$$
D_{i}=\epsilon_{i j} E_{j} \quad i, j=1, \ldots, 6 .
$$

For an anisotropic medium, the energy density of the electric field stored in the medium is:

$$
U_{e}=\frac{1}{2} E \cdot D=\frac{1}{2} E_{i} \epsilon_{i j} E_{j} .
$$

Surfaces of energy density $U_{e}$, which are constant in the space defined by the dielectric tensor $D$, can be described by the following equation:

$$
\frac{D_{x_{1}}^{2}}{\epsilon_{x_{1}}}+\frac{D_{x_{2}}^{2}}{\epsilon_{x_{2}}}+\frac{D_{x_{3}}^{2}}{\epsilon_{x_{3}}}=2 U_{e}
$$

where $\epsilon_{x_{1}}, \epsilon_{x_{2}}$, and $\epsilon_{x_{3}}$ are the principal dielectric constants.

The principal refractive indices $n_{x_{1}}, n_{x_{2}}$, and $n_{x_{3}}$ are defined by $n_{x_{i}}^{2}=\epsilon_{i} / \epsilon_{0}$. By substituting $D / \sqrt{2 U_{e}}$ with the position vector $r$, Eq. (A.4) gives:

$$
\frac{x_{1}^{2}}{n_{x_{1}}^{2}}+\frac{x_{2}^{2}}{n_{x_{2}}^{2}}+\frac{x_{3}^{2}}{n_{x_{3}}^{2}}=1
$$


The last equation (A.5) is that of an ellipsoid with its main axes parallel to $x_{1}, x_{2}$, and $x_{3}$ axes and with respective lengths of $2 n_{x_{1}}, 2 n_{x_{2}}$, and $2 n_{x_{3}}$. This ellipsoid is called Fresnel Ellipsoid or Refractive Index Ellipsoid. It is mainly used to find the two refraction indices and the two directions of the dielectric tensor $D$ associated with the two plane waves that can be propagated along an arbitrary direction $s$ of the medium.

The photoelastic effect representing the refractive index variations of a material subjected to a strain field can be expressed by:

$$
\Delta \eta_{i j}=\Delta\left(\frac{1}{n^{2}}\right)_{i j}=p_{i j k l} \varepsilon_{k l}
$$

with $\Delta \eta_{i j}$ the permittivity tensor variation defined by $\eta_{i j}=\epsilon_{0}\left(\epsilon^{-1}\right)_{i j}$, where $\epsilon^{-1}$ is the inverse of the dielectric tensor $\epsilon$; $\varepsilon_{k l}$ the strain tensor; and $p_{i j k l}$ the tensor of the photoelastic constants (in $B \mathrm{w})$.

The Fresnel ellipsoid for a material subjected to a strain field is then given by the equation:

$$
\left(\eta_{i j}+p_{i j k l} \varepsilon_{k l}\right) x_{i} x_{j}=1 .
$$

When brought back into the reference frame and also because of the tensors' symmetry, Eq. (A.6) leads to, with contracted notations:

$$
\Delta\left(\frac{1}{n^{2}}\right)_{i}=p_{i j} \varepsilon_{j} \quad i, j=1, \ldots, 6 .
$$

Thus, the Fresnel ellipsoid submitted to a strain field is defined by:

$$
\begin{aligned}
& x_{1}^{2}\left(\frac{1}{n_{x_{1}}^{2}}+p_{11} \varepsilon_{1}+p_{12} \varepsilon_{2}+p_{13} \varepsilon_{3}+p_{14} \varepsilon_{4}+p_{15} \varepsilon_{1}+p_{16} \varepsilon_{6}\right) \\
+ & x_{2}^{2}\left(\frac{1}{n_{x_{2}}^{2}}+p_{21} \varepsilon_{1}+p_{22} \varepsilon_{2}+p_{23} \varepsilon_{3}+p_{24} \varepsilon_{4}+p_{25} \varepsilon_{1}+p_{26} \varepsilon_{6}\right) \\
+ & x_{3}^{2}\left(\frac{1}{n_{x_{3}}^{2}}+p_{31} \varepsilon_{1}+p_{32} \varepsilon_{2}+p_{33} \varepsilon_{3}+p_{34} \varepsilon_{4}+p_{35} \varepsilon_{1}+p_{36} \varepsilon_{6}\right) \\
+ & 2 x_{1} x_{2}\left(p_{41} \varepsilon_{1}+p_{42} \varepsilon_{2}+p_{43} \varepsilon_{3}+p_{44} \varepsilon_{4}+p_{45} \varepsilon_{1}+p_{46} \varepsilon_{6}\right) \\
+ & 2 x_{2} x_{3}\left(p_{51} \varepsilon_{1}+p_{52} \varepsilon_{2}+p_{53} \varepsilon_{3}+p_{54} \varepsilon_{4}+p_{55} \varepsilon_{1}+p_{56} \varepsilon_{6}\right) \\
+ & 2 x_{1} x_{3}\left(p_{61} \varepsilon_{1}+p_{62} \varepsilon_{2}+p_{63} \varepsilon_{3}+p_{64} \varepsilon_{4}+p_{65} \varepsilon_{1}+p_{66} \varepsilon_{6}\right) \\
= & 1
\end{aligned}
$$

where $n_{x_{1}}, n_{x_{2}}$, and $n_{x_{3}}$ are the principal refractive indices.

\section{Appendix A.2. Application to the single-mode optical fiber}

The light transmitted by a single-mode FO can be split up into two linearly polarized terms. If the central axis of the fiber is directed along the $x_{1}$-axis (Fig. 11), the two polarized components of the light transmitted in the single-mode FO are orthogonal and propagated in the planes $\left(x_{1}\right.$, $\left.x_{2}\right)$ and $\left(x_{1}, x_{3}\right)$. These two components make it possible to calculate the average refractive index of each point of the fiber by calculating the average of their respective refractive indices $n_{x_{2}}$ and $n_{x_{3}}: n=\left(n_{x_{2}}+n_{x_{3}}\right) / 2$.

[Figure 11 about here.] 
The refractive indexes $n_{x_{2}}$ and $n_{x_{3}}$ can be directly calculated from Eq. (A.8):

$$
\left\{\begin{array}{l}
\Delta\left(\frac{1}{n^{2}}\right)_{2}=p_{2 j} \varepsilon_{j} \\
\Delta\left(\frac{1}{n^{2}}\right)_{3}=p_{3 j} \varepsilon_{j}
\end{array}\right.
$$

so:

$$
\left\{\begin{array}{l}
\frac{1}{n_{x_{2}}^{2}}=\frac{1}{n_{0}^{2}}+p_{2 j} \varepsilon_{j} \\
\frac{1}{n_{x_{3}}^{2}}=\frac{1}{n_{0}^{2}}+p_{3 j} \varepsilon_{j}
\end{array}\right.
$$

hence:

$$
\left\{\begin{array}{l}
n_{x_{2}}^{2}=\frac{n_{0}}{\sqrt{1+n_{0}^{2} p_{2 j} \varepsilon_{j}}} \\
n_{x_{3}}^{2}=\frac{n_{0}}{\sqrt{1+n_{0}^{2} p_{3 j} \varepsilon_{j}}}
\end{array} .\right.
$$

For a single-mode optical fiber, the expression of the average refractive index is [25]:

$$
n=\frac{n_{0}}{2}\left(\frac{1}{\sqrt{1+n_{0}^{2} p_{2 j} \varepsilon_{j}}}+\frac{1}{\sqrt{1+n_{0}^{2} p_{3 j} \varepsilon_{j}}}\right) \quad i, j=1, \ldots, 6
$$

where $n_{0}$ is the constant part of the refractive index and $p_{2 j}$ and $p_{3 j}$ are the terms of the photoelastic constant tensor.

\section{Appendix A.3. Influence of the optical fiber sheath}

The strain transfer from the host material to the optical fiber core will depend on the CALGFOS sheath and its connection to the structure: either glued or cored. Indeed, the effectiveness of the structural strain transfer to the sensor will depend on the selected sheath. A tensor is thus introduced to take strain transfer into account. Systematic shifts of about $10 \%$ can be observed for axial strain. In addition, the axial components of the tensor may not be constant along the optical fiber [15, 23]. Strain restitution modeling is not proposed here except where this effect would intervene in equations. Then the material strain transmitted to the sensor can be modeled by $\varepsilon^{1}=\beta \cdot \varepsilon^{0}$, where $\varepsilon^{1}$ is the strain in the optical fiber induced by the host material, $\varepsilon^{0}$ the strain applied on the host material, and $\beta$ the strain transfer tensor.

\section{Appendix A.4. Temperature influence}

Temperature will influence both the material of the studied structure and the silica of the optical fiber. For the structure being studied, the additional term due to temperature variation in the calculation of the curvilinear abscissa variation $d s$ defined in Eq. (5) is:

$$
d s_{T}=\alpha_{i j} \Delta T d x_{i} d x_{j}
$$

where $\alpha_{i j}$ are the thermal expansion tensor components (in $K^{-1}$ or ${ }^{\circ} C^{-1}$ ) and $\Delta T=T-T_{0}$ is the temperature variation compared to the initial temperature.

For the CA-LGFOS, the temperature effect is double: first on the refractive index and second on the optical fiber strain. For the refractive index, the additional term due temperature variation is:

$$
n_{T}=\frac{\partial n}{\partial T} \Delta T
$$


where $\partial n / \partial T$ is the refractive index dependence on temperature, whose experimental determination is given in [31]. Since optical fiber is assumed to be an isotropic material, thermal expansion is the same in all directions.

For the fiber constituting the sensor, temperature variation will induce strain. The optical fiber lengthening due to temperature variation is defined by:

$$
\Delta L_{\text {phys }}^{T}(a, b, t)=\alpha_{0}(b-a) \Delta T
$$

where $\Delta L_{\text {phys }}^{T}(a, b, t)=L_{\text {phys }}^{T}(a, b, t)-L_{\text {phys }}^{T_{0}}(a, b, 0)$, with $L_{\text {phys }}^{T_{0}}(a, b, 0)$ being the initial physical length of the CA-LGFOS and $\alpha_{0}$ the thermal expansion coefficient of the optical fiber.

This thermal expansion is called free thermal expansion, as previously defined in Eq. (A.14). However, when FOS is inserted into a material, thermal expansions of the material and those of the various components of the sensor (core, primary, and secondary coatings) interact. The various thermal expansions can then be constrained $[40,41]$. An interactions modeling is not proposed here. However, we indicate where this effect intervenes in equations. To take into account interactions between the incorporated optical fiber and the thermal expansions of the host material, the use of a tensor $\alpha_{i j}^{i n t}$ is proposed instead of the thermal expansion tensor for the curvilinear abscissa variation defined in Eq. (A.14), so that:

$$
d s^{2}=\left(g_{i j}+2 \varepsilon_{i j}+\alpha_{i j}^{i n t}\right) d x_{i} d x_{j} .
$$

\section{Appendix A.5. CA-LGFOS measurement expression}

By gathering the influence of various parameters (strain, temperature, and coating), the measurement of CA-LGFOS is defined as, with contracted notations:

$L_{\text {opt }}\left(s_{a}, s_{b}, t\right)=\int_{s_{a}}^{s_{b}}\left[\frac{n_{0}}{2}\left(\frac{1}{\sqrt{1+n_{0}^{2} p_{2 k} \beta_{k}\left(\varepsilon_{k}+\alpha_{k}^{\text {int }} \Delta T\right)}}+\frac{1}{\sqrt{1+n_{0}^{2} p_{3 k} \beta_{k}\left(\varepsilon_{k}+\alpha_{k}^{\text {int }} \Delta T\right)}}\right)+\frac{\partial n}{\partial T} \Delta T\right] d s$

where $d s=\sqrt{\left[g_{k}+2 \beta_{k}\left(\varepsilon_{k}+\alpha_{k}^{i n t} \Delta T\right)\right] d x_{k}}$ and $k=1, \ldots, 6$.

\section{References}

[1] A. Alvandi, C. Cremona, Assessment of vibration-based damage identification techniques, Journal of Sound and Vibration 292 (2006) 179-202.

[2] W.X. Ren, X.L. Peng, Y.Q. Lin, Experimental and analytical studies on dynamic characteristics of a large span cable-stayed bridge, Engineering Structures 27 (2005) 535-548.

[3] M. Meo, G. Zumpano, On the optimal sensor placement techniques for a bridge structure, Engineering Structures 27 (2005) 1488-1497.

[4] T.H. Yan, R.M. Lin, General optimization of sizes or placement for various sensors/actuators in structure testing and control, Smart Materials and Structures 15 (2006) 724-736.

[5] D. Inaudi, A. Elamari, L. Pflug, N. Gisin, J. Breguet, S. Vurpillot, Low-coherence deformation sensors for the monitoring of civil-engineering structures, Sensors and Actuators A 44 (1994) 125-130.

[6] Y. Zhao, F. Ansari, Quasi-distributed fiber-optic strain sensor: principle and experiment, Applied Optics 40 (2001) 3176-3181.

[7] J.M. Lopez-Higuera, Handbook of Optical Fibre Sensing Technology, Wiley \& Sons, New York, 2002. 
[8] J.R. Dunphy, G. Meltz, W.W. Morey, Optical Fiber Bragg Grating Sensors : A Candidate for Smart Structure Applications, Wiley \& Sons, Fiber Optic Smart Structures, New York, pp. 271-285.

[9] J.G. Liu, C. Schmidt-Hattenberg, G. Borm, Dynamic strain measurement with a fibre Bragg grating sensor system, Measurement 32 (2002) 151-161.

[10] P. Capoluongo, C. Ambrosinoa, S. Campopianoa, A. Cutoloa, M. Giordanob, I. Bovioc, L. Leccec, A. Cusano, Modal analysis and damage detection by Fiber Bragg grating sensors, Sensors and Actuators A 133 (2006) 415-424.

[11] B. Glisic, D. Inaudi, Fibre Optic Methods for Structural Health Monitoring, Wiley \& Sons, Chichester, England, 2007.

[12] A. Del Grosso, A. Torre, D. Inaudi, G. Brunetti, A. Pietrogrande, Monitoring System for a Cable-Stayed Bridge using static and dynamic Fiber Optic Sensors, Second International Conference on Structural Health Monitoring of Intelligent Infrastructure, November 16-18, Shenzhen, China, 2005, pp. 415-420.

[13] D. Inaudi, S. Vurpillot, Monitoring of Concrete Bridges with Long-Gage Fiber Optic Sensors, Journal of Intelligent Material Systems and Structures 10 (1999) 280-292.

[14] D. Inaudi, B. Glisic, Combining Static and Dynamic deformation monitoring with long-gauge fiber optic sensors, International Association for Bridge Maintenance and Safety, October 19-22, Kyoto, Japan, 2004.

[15] S. Delepine-Lesoille, E. Merliot, C. Boulay, L. Quétel, M. Delaveau, A. Courteville, Quasi-distributed optical fibre extensometers for continuous embedding into concrete: design and realization, Smart Materials and Structures 15 (2006) 931-938.

[16] B. Glisic, D. Inaudi, Integration of long-gage fiber-optic sensor into a fiber-reinforced composite sensing tape, International Symposium on Smart Structures and Materials, March 3-7, San Diego, USA, 2003, Vol. 5050-28 pp. $179-186$.

[17] E. Reynders, G. De Roeck, Measurement of modal curvature using optical fiber strain sensors and application to damage identification using vibration monitoring, Seventeenth International Conference on Optical Fiber Sensors, May 23-27, Bruge, Belgium, 2005, SPIE Vol. 5855 pp. 1076-1079.

[18] F. Casciati, M. Domaneschi, D. Inaudi, A. Figini, B. Glisic, A. Gupta, Long-gauge fiber optic sensors: a new approach to dynamic system identification, Third European Conference on Structural Control, July 12-15, Vienna, Austria, 2004.

[19] S. Delepine-Lesoille, E. Merliot, Y. Gautier, L. Quetel, M. Delaveau, A. Courteville, Multiplexed Long-Base Flexible Optical Fiber Extensometers and Temperature Bragg Sensors Interrogated by Low-Coherence Interferometry, IEEE Sensors Journal 8 (2008) 1145-1151.

[20] Y. Zhao, F. Ansari, Quasi-distributed white light fiber optic strain sensor, Optics communication 196 (2001) 133-137.

[21] J.P. Perez, Optique: fondements et applications avec 250 exercices et problèmes résolus [in French], Dunod, Paris, 2004.

[22] C.D. Butter, G. B. Hocker, Fiber optics strain gauge, Applied Optics 17(18) (1978) 2867-2869.

[23] F. Ansari, L. Yuan, Mechanics of Bond and Interface Shear Transfer in Optical Fiber Sensors, Journal of Engineering Mechanics 124 (1998) 385-394.

[24] L. Yuan, J. Yang, Multiplexed MachZehnder and Fizeau tandem white light interferometric fiber optic strain/temperature sensing system, Sensors and Actuators A 105(1) (2003) 40-46.

[25] J.S. Sirkis, H.W. Haslach, Interferometric Strain Measurement by Arbitrarily Configured Surface-Mounted Optical Fibers, Journal of Lightwave Technology 10 (1990) 1497-1503.

[26] T.G. Giallorenzi, J.A. Bucaro, A. Dandridge, G.H. Sigel Jr, J.H. Cole, S.C. Rashleigh, R.G. Priest, Optical Fiber Sensor Technology, Journal of Quantum Electronics 4 (1982) 626-665.

[27] Z. Chen, Q. Li, F. Ansari, Serial Multiplexing of Optical Fibers for Sensing of Structural Strains, Journal of Structural Control 7 (2000) 103-117.

[28] K. Tian, Y. Liu, Q. Wang, Temperature-independent fiber Bragg grating strain sensor using bimetal cantilever Original Research Article, Optical Fiber Technology 11(4) (2005) 370-377.

[29] M. Pozzi, D. Zonta, H. Wu, D. Inaudi, Development and laboratory validation of in-line multiplexed lowcoherence interferometric sensors, Optical Fiber Technology 14 (2008) 281-293.

[30] B. Liu, H. Zhang, Polarimetric distributed Bragg reflector fiber laser sensor array for simultaneous measurement of transverse load and temperature, Optical Fiber Technology 17(6) (2011) 619-625.

[31] D. Williams, J. Carr, S. Saikkonen, Single-mode optical fiber index of refraction dependence on product parameters, tensile stress and temperature, Thirty ninth International Wire and Cable Symposium, November 13-15, Reno, USA, 1990, pp. 726-729.

[32] M. Geradin, D. Rixen, Mechanical Vibrations: Theory and Applications to Structural Dynamics, Wiley \& Sons, 
1997.

[33] S. Vurpillot, G. Krueger, D. Benouaich, D. Clément, D. Inaudi, Vertical deflection of a pre-stressed concrete bridge obtained using deformation sensors and inclinometer measurements, ACI Structural Journal 95 (1998) 518-526.

[34] D. Zonta, M. Pozzi, M. Forti, P. Zanon, Vibration-based condition monitoring of smart prefabricated concrete elements, Key Engineering Materials 293 (2005) 743-750.

[35] T.P. Le, P. Argoul, Continuous wavelet transform for modal identification using free decay response, Journal of Sound and Vibration 277 (2004) 73-100.

[36] P. Argoul, T.P. Le, Wavelet analysis of transient signals in civil engineering, Novel Approaches in Civil Engineering 14 (2004) 311-318.

[37] S. Calvert, J.P. Conte, B. Moaveni, W.L. Schulz, R. de Callalfon, Real time damage assessment using fiber optic grating sensors, Sixth Pacific Northwest Fiber Optic Sensor Workshop, May 14-15, Troutdale, USA, 2003, SPIE Vol. 5278 pp. $110-121$.

[38] M.M. Abdel Wahab, G. De Roeck, Damage detection in bridges using modal curvatures: application to a real damage scenario, Journal of Sound and Vibration 226(2) (1999) 217-235.

[39] A. Yariv, P. Yeh, Optical Waves in Crystals Propagation and Control of Laser Radiation, Wiley \& Sons, New York, 2003.

[40] M.J. LeBlanc, Study of interfacial interaction of an optical fibre embedded in a host material by in situ measurement of fibre end displacement - Part 1. Theory, Smart Materials and Structures 14 (2001) 637-646.

[41] M.J. LeBlanc, Study of interfacial interaction of an optical fibre embedded in a host material by in situ measurement of fibre end displacement - Part 2. Experiments, Smart Materials and Structures 14 (2001) 647-657.

\section{Biographies}

Gwendal Cumunel graduated as a mechanical engineer from SUPMECA Paris with a specialization in Dynamics. He wrote his $\mathrm{PhD}$ thesis in applied mechanics about the use of fiber optic long-gage optical fiber extensometers for the dynamic evaluation of structures. He works as a researcher at NAVIER Laboratory in the Structural Dynamics and Identification Group. His main research topics concern the modeling and identification of linear and nonlinear mechanical structures under vibration, as well as extensometry in dynamics, especially as it relates to long-gage optical fiber sensors.

Sylvie Delepine-Lesoille graduated as an engineer from Ecole Nationale Supérieure de Techniques Avancées with a specialization in Nonlinear Optics. She joined Alcatel Corporate Research Center as a PhD candidate to work with the semiconductor flared laser. Next, she worked for two years at Photonetics-NetTest on tunable and wavelength switchable external cavity lasers. Then for seven years, she was a researcher in the division of Metrology and Instrumentation of LCPC (French Road and Bridges Institute) on optical fiber sensors for civil engineering applications. She works at ANDRA on the instrumentation of nuclear waste geological repository, especially as it relates to optical fiber sensors. She is the French representative for COST action OFSeSa. Over 20 of her research papers have been published.

Pierre Argoul graduated as a civil engineer at Ecole Nationale des Travaux Publics de l'Etat and wrote his $\mathrm{PhD}$ thesis in applied mechanics. He is authorized to supervise PhD theses. He works as a research director at NAVIER Laboratory as well as an assistant professor at Ecole Nationale des Ponts et Chaussées. His main research topics are about the modeling and identification of linear and nonlinear mechanical structures under vibration or wave propagation. Some recent applications include the damping effect in structures and material, damage in glued structural connections, and crowd movements. He is also an expert in time-frequency transforms for the processing of signals and systems. Over 80 of his research papers have been published. 


\section{List of Figures}

1 Fiber Optic Sensor from IDIL Fibres Optiques. . . . . . . . . . . . . . . . . . 21

2 Measurement principle of FOGALE nanotech optical device. . . . . . . . . . . . 22

3 Application case - Straight CA-LGFOS on a slender beam. . . . . . . . . . . . . 23

4 CWT absolute value of the numerical signals from the different measurement types. 24

5 Clamped-clamped beam - Observed mode shapes obtained through the CWT method with CA-LGFOS and standard punctual sensor numerical signals. . . . . . . . . . 25

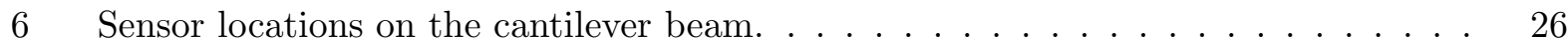

$7 \quad$ Photography of the instrumented cantilever beam. . . . . . . . . . . . . . 27

8 Cantilever beam - Signals and corresponding FFT obtained with accelerometer $n^{\circ} 8$, strain gauge $n^{\circ} 1$, and CA-LGFOS $n^{\circ} 1 \ldots \ldots \ldots \ldots \ldots$

9 Cantilever beam - Observed mode shapes obtained through the CWT method with the measurement signals of the accelerometers, strain gauges, and CA-LGFOS. . . 29

10 Cantilever beam - Instantaneous frequencies obtained through the CWT method with the measurement signals of the accelerometers, strain gauges, and CA-LGFOS. 30

11 Configuration of the single-mode FO . . . . . . . . . . . . . . . . 31 


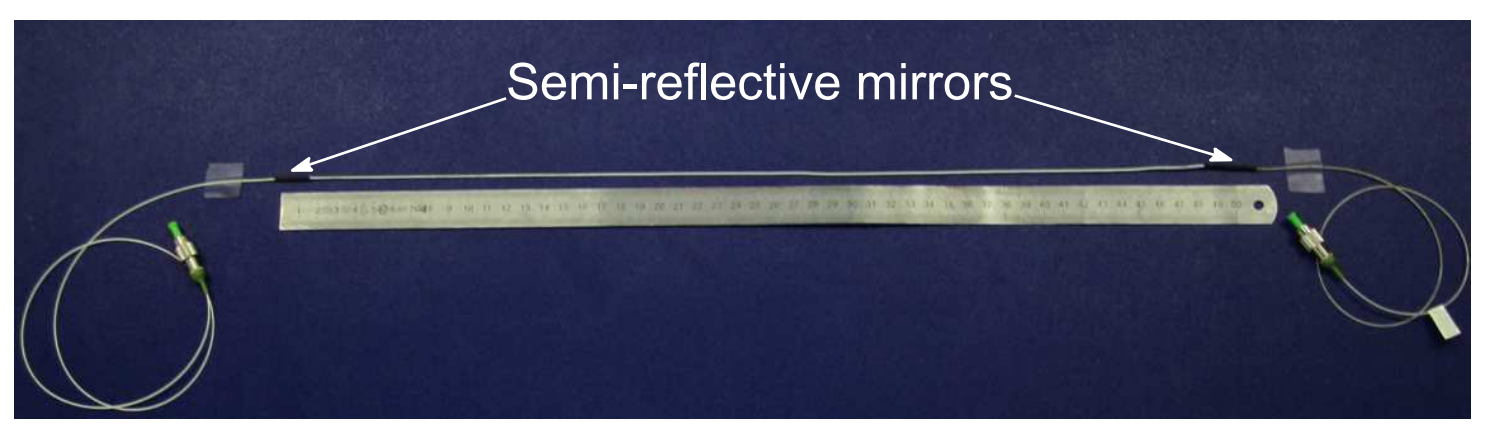

(a) Sheathed sensor with a $50 \mathrm{~cm}$ gage length.

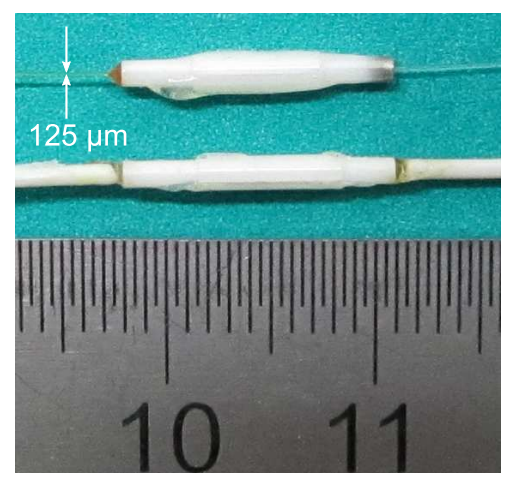

(b) Semi-reflective mirror in ferrule with sheathed and unsheathed optical fiber.

Figure 1: Fiber Optic Sensor from IDIL Fibres Optiques. 


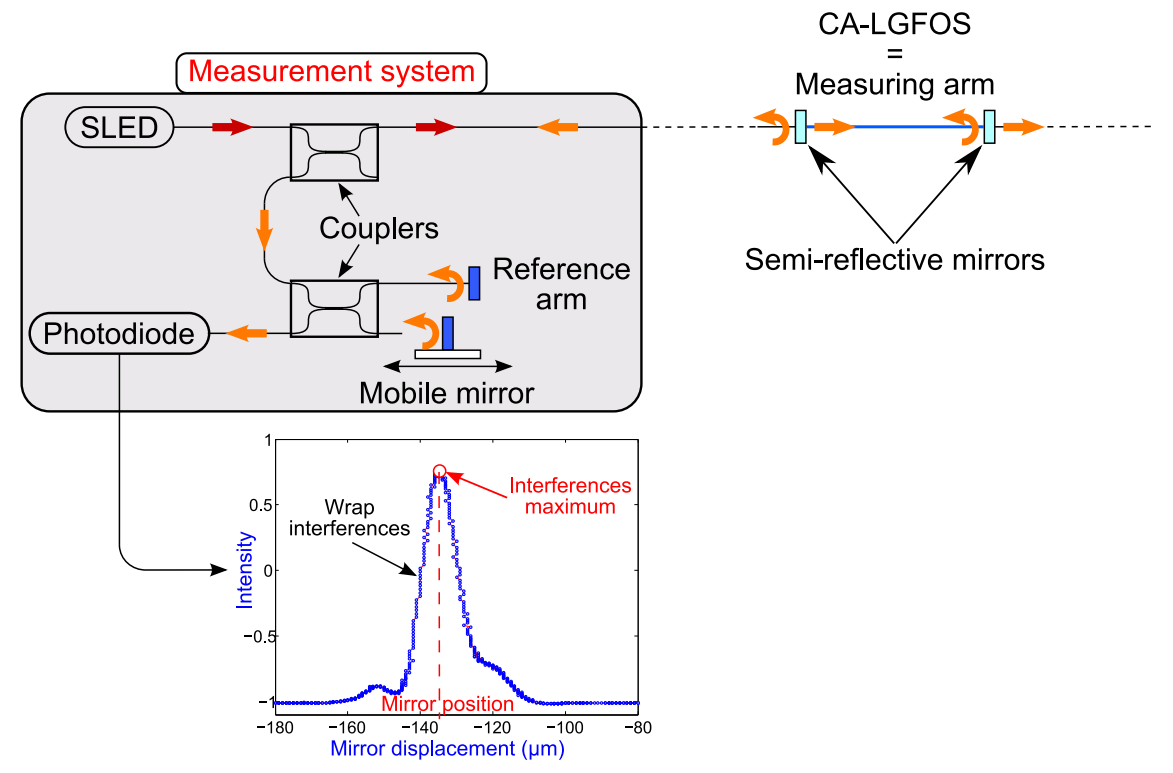

Figure 2: Measurement principle of FOGALE nanotech optical device. 


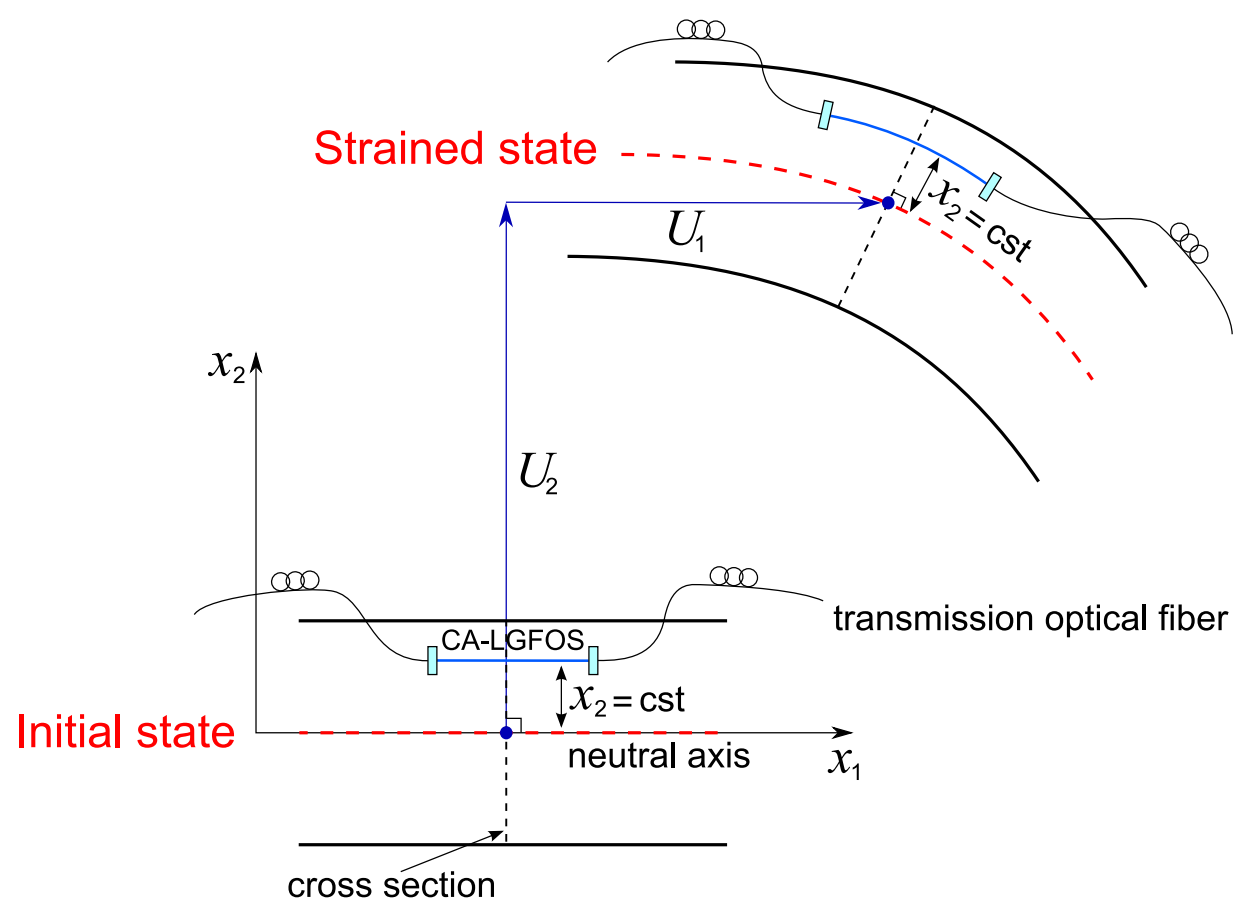

Figure 3: Application case - Straight CA-LGFOS on a slender beam. 


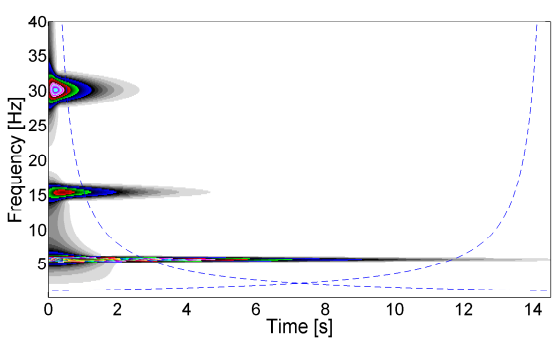

(a) Displacement $v$

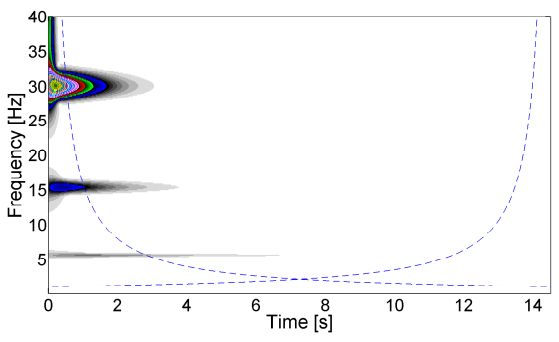

(c) Acceleration $\ddot{v}$

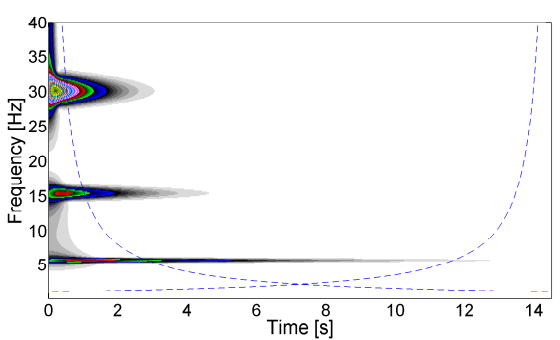

(b) Velocity $\dot{v}$

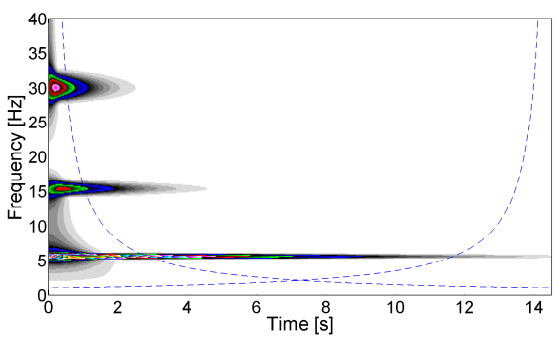

(d) CA-LGFOS

Figure 4: CWT absolute value of the numerical signals from the different measurement types. 


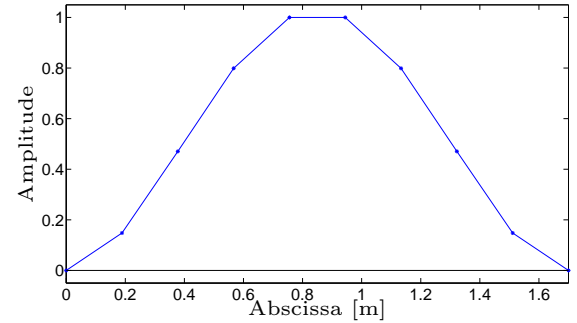

(a) Punctual sensors - mode shape $n^{\circ} 1$

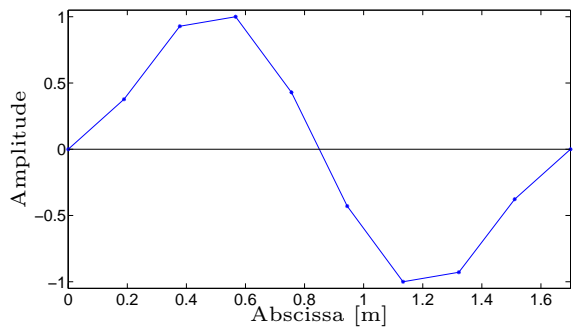

(c) Punctual sensors - mode shape $n^{\circ} 2$

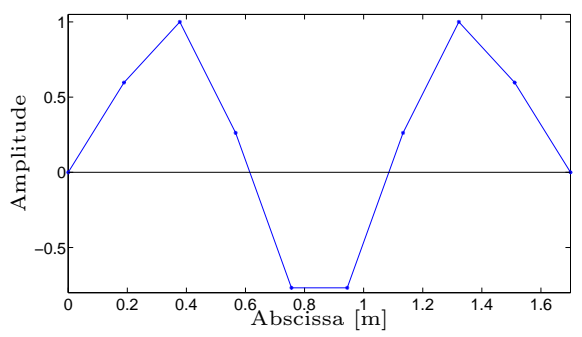

(e) Punctual sensors - mode shape $n^{\circ} 3$

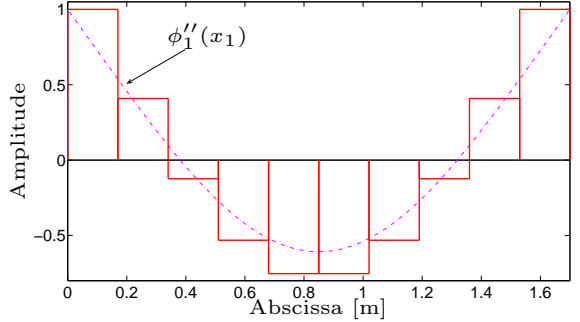

(b) CA-LGFOS - curvature mode shape $n^{\circ} 1$

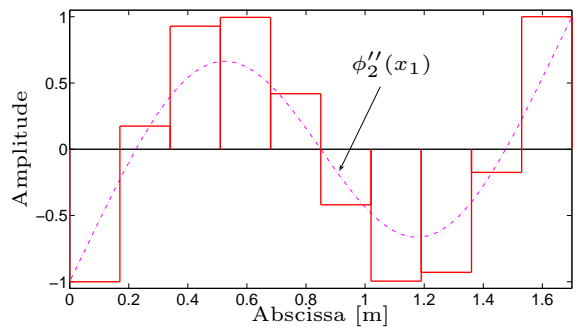

(d) CA-LGFOS - curvature mode shape $n^{\circ} 2$

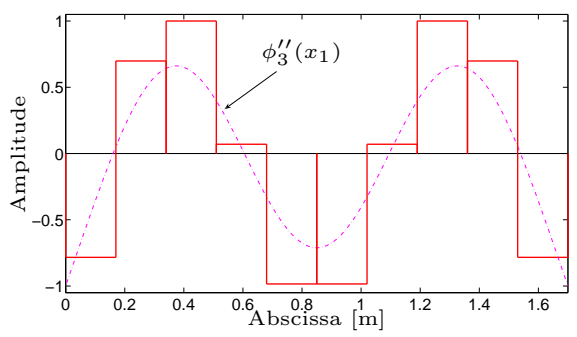

(f) CA-LGFOS - curvature mode shape $n^{\circ} 3$

Figure 5: Clamped-clamped beam - Observed mode shapes obtained through the CWT method with CA-LGFOS and standard punctual sensor numerical signals. 

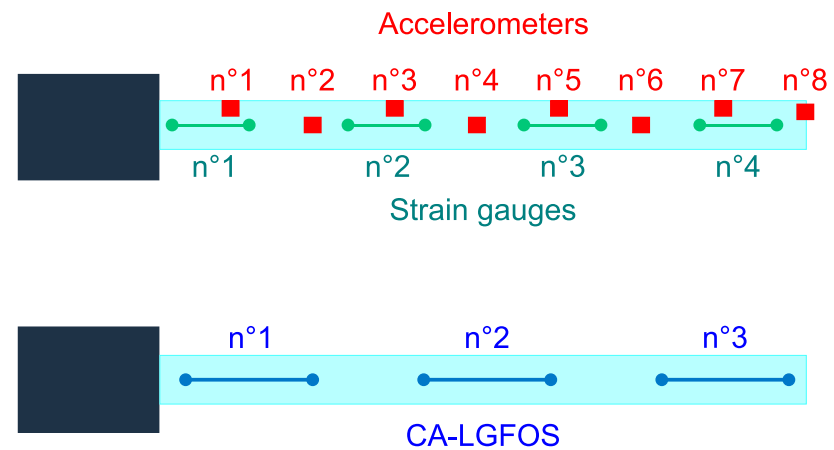

Figure 6: Sensor locations on the cantilever beam. 


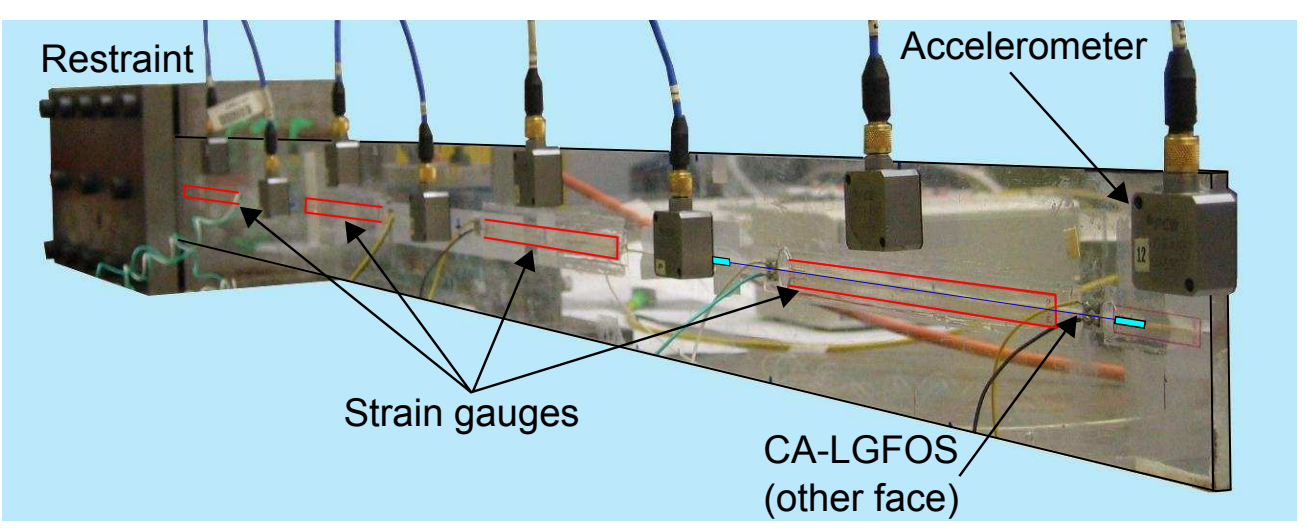

Figure 7: Photography of the instrumented cantilever beam. 


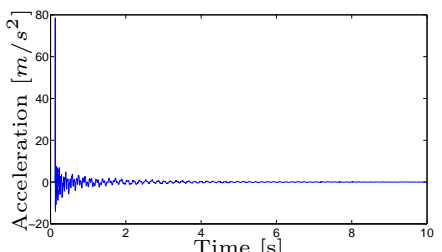

(a) Accelerometer $\mathrm{n}^{\circ} 8$ signal

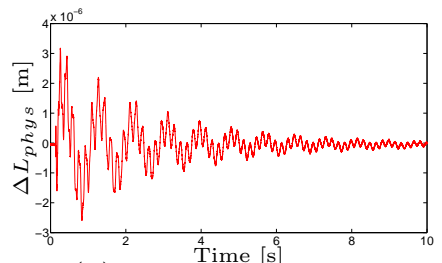

(d) Strain gauge $\mathrm{n}^{\circ} 1$ signal

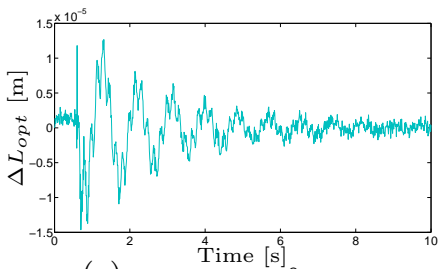

(g) CA-LGFOS $\mathrm{n}^{\circ} 1$ signal

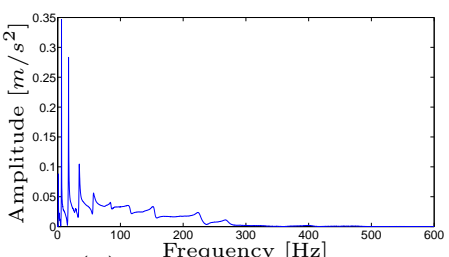

(b) Accelerometer $\mathrm{n}^{\circ} 8 \mathrm{FFT}$

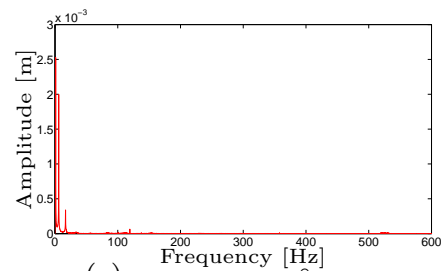

Frequency $[\mathrm{Hz}]$
(e) Strain gauge $\mathrm{n}^{\circ} 1 \mathrm{FFT}$

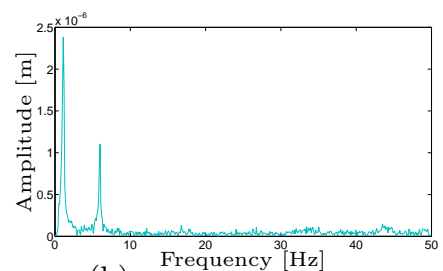

(h) CA-LGFOS n ${ }^{\circ} 1$ FFT

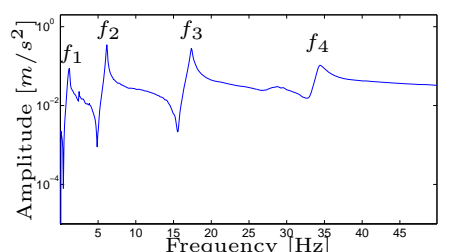

(c) Accelerometer $n^{\circ} 8$ FFT zoom $+y-\log$

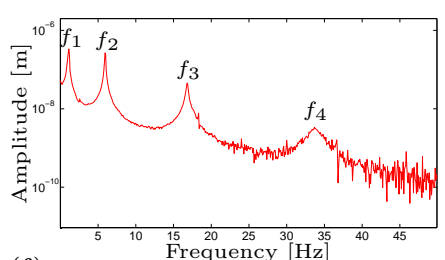

Frequency $[\mathrm{Hz}]$
(f) Strain Gauge n ${ }^{\circ} 1 \mathrm{FFT}^{\mathrm{F}}$ zoom $+\mathrm{y}$-log

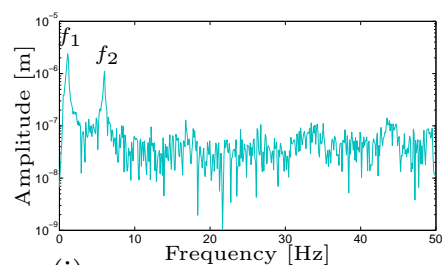

Frequency $[\mathrm{Hz}]$
(i) CA-LGFOS n ${ }^{\circ} 1 \mathrm{FFT}+\mathrm{y}-\mathrm{log}$

Figure 8: Cantilever beam - Signals and corresponding FFT obtained with accelerometer $\mathrm{n}^{\circ} 8$, strain gauge $\mathrm{n}^{\circ} 1$, and CA-LGFOS $n^{\circ} 1$. 


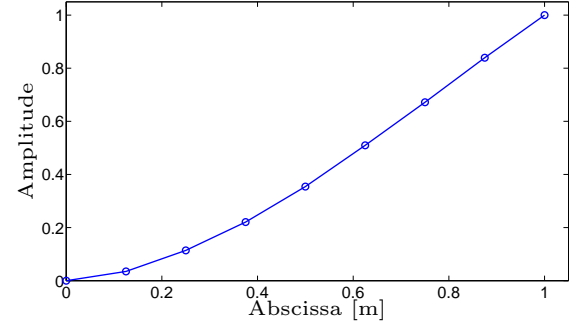

(a) Accelerometers - observed mode shape $n^{\circ} 1$

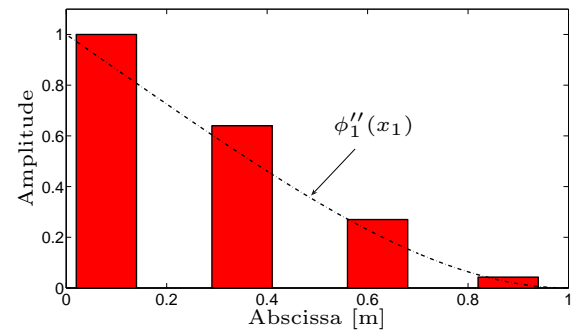

(c) Strain gauges - observed mode shape $\mathrm{n}^{\circ} 1$

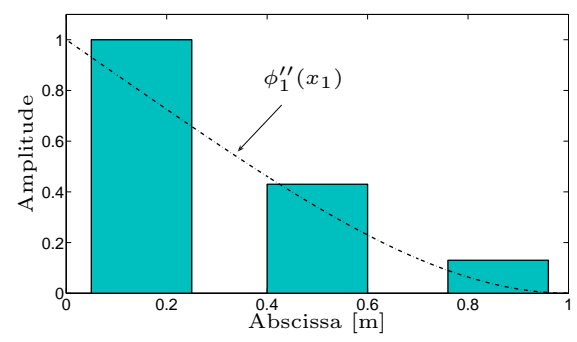

(e) CA-LGFOS - observed mode shape $n^{\circ} 1$

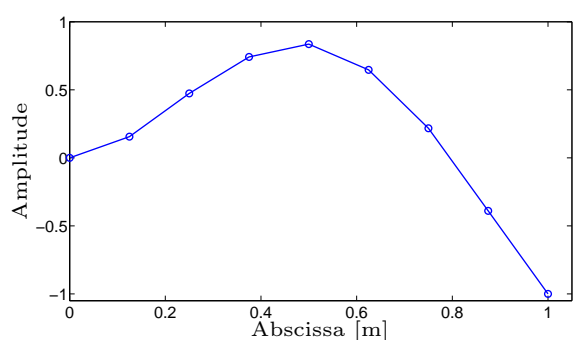

(b) Accelerometers - observed mode shape $\mathrm{n}^{\circ} 2$

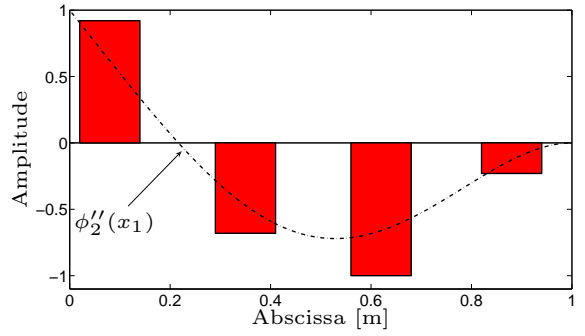

(d) Strain gauges - observed mode shape $\mathrm{n}^{\circ} 2$

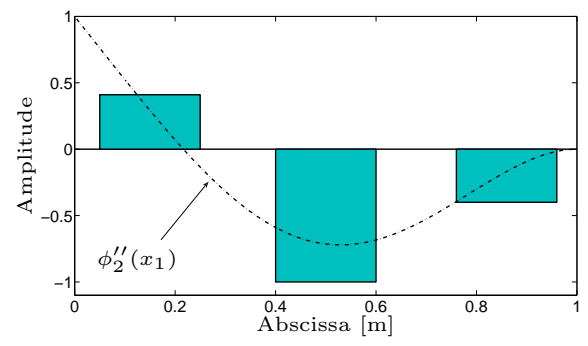

(f) CA-LGFOS - observed mode shape $\mathrm{n}^{\circ} 2$

Figure 9: Cantilever beam - Observed mode shapes obtained through the CWT method with the measurement signals of the accelerometers, strain gauges, and CA-LGFOS. 


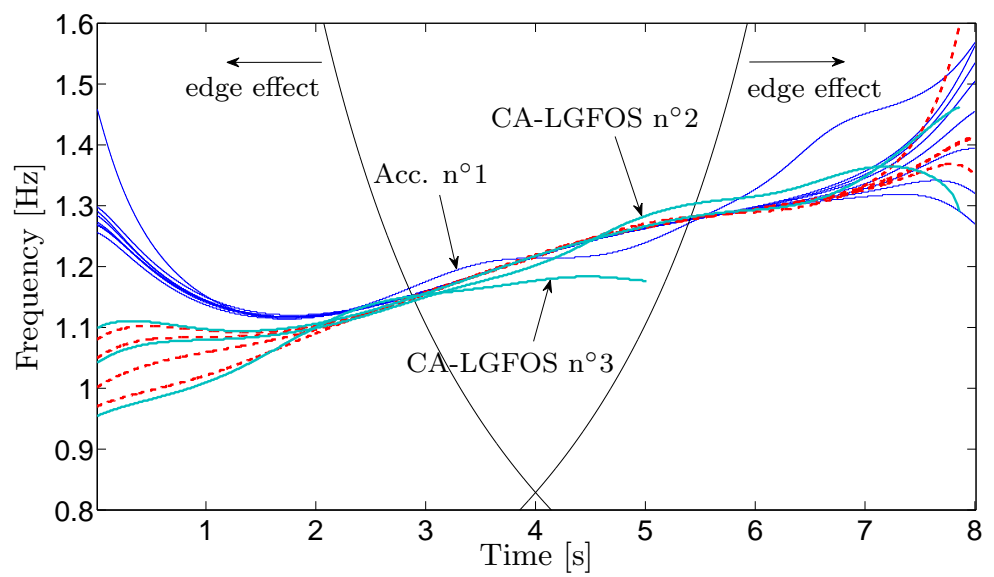

(a) First mode (Acc.: blue lines, Gauge: dashed red lines, CA-LGFOS: bold lines)

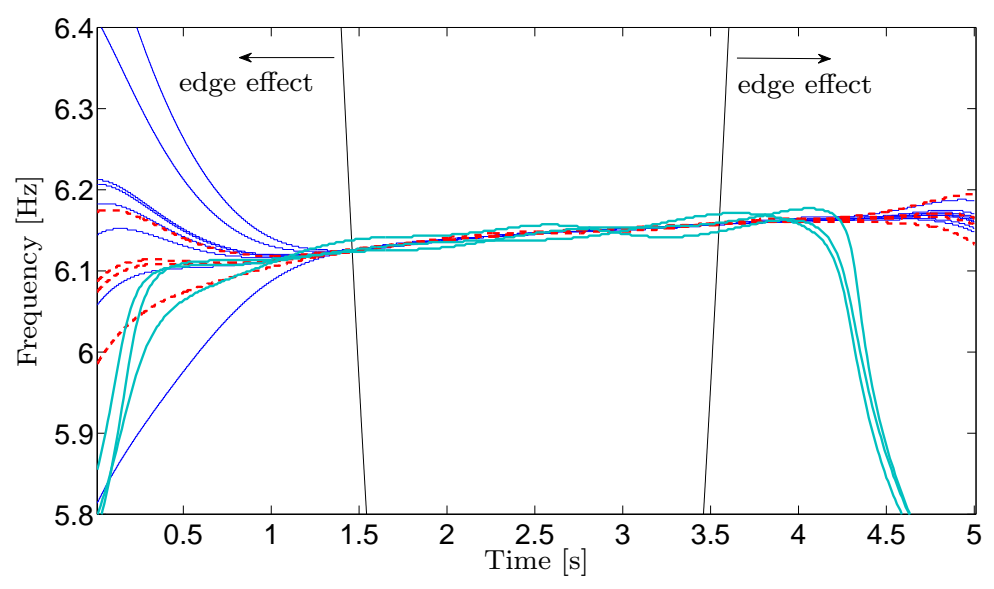

(b) Second mode (Acc.: blue lines, Gauge: dashed red lines, CA-LGFOS: bold lines)

Figure 10: Cantilever beam - Instantaneous frequencies obtained through the CWT method with the measurement signals of the accelerometers, strain gauges, and CA-LGFOS. 


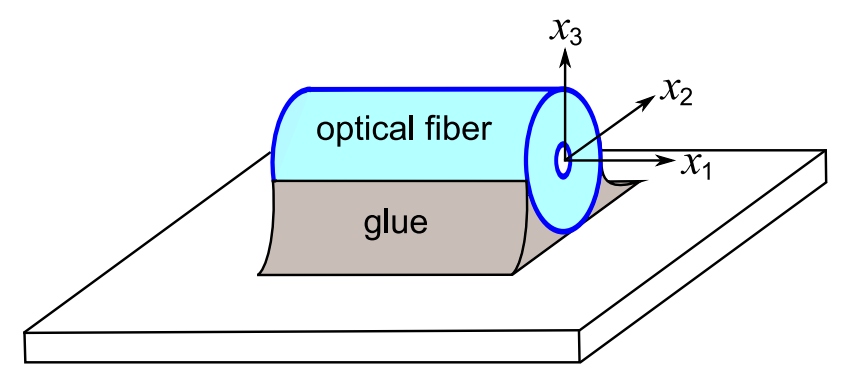

Figure 11: Configuration of the single-mode FO. 


\section{List of Tables}

1 Clamped-clamped beam characteristics. . . . . . . . . . . . . . . . . . 33

2 Numerical simulation parameters. . . . . . . . . . . . . . . . . . . 34

3 CA-LGFOS and standard punctual sensor locations on the clamped-clamped beam. 35

4 Parameters of CWT method for local treatment of numerical signals from different

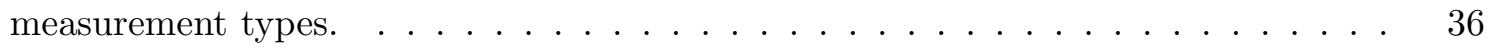

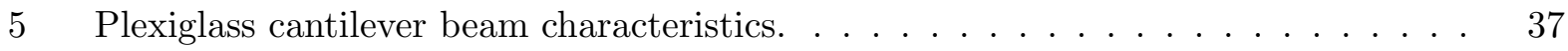

6 CA-LGFOS, accelerometer, and gauge locations on the cantilever beam. . . . . . 38

7 Eigenfrequencies and modal damping ratios obtained by peak picking and CWT methods for the first and second modes of the cantilever beam with the accelerometers, strain gauges, and CA-LGFOS. . . . . . . . . . . . . .

8 Eigenvectors obtained by peak picking and CWT methods for the first and second modes of the cantilever beam with the accelerometers, strain gauges, and CA-LGFOS. 40 


\begin{tabular}{cccccc}
\hline Length & Thickness & Height & Young modulus & Density & Inertia \\
\hline$L(\mathrm{~m})$ & $e(\mathrm{~m})$ & $h(\mathrm{~m})$ & $E_{0}(\mathrm{GPa})$ & $\rho\left(\mathrm{kg} \cdot \mathrm{m}^{-3}\right)$ & $I\left(\mathrm{~m}^{4}\right)$ \\
1.7 & 0.04 & 0.003 & 210 & 7800 & $9 \cdot 10^{-11}$ \\
\hline
\end{tabular}

Table 1: Clamped-clamped beam characteristics. 


\begin{tabular}{cccccc}
\hline Shock location & Shock time & Final time & Points nb & Damping ratio & Modes nb \\
\hline$x_{0}(\mathrm{~m})$ & $t_{0}(\mathrm{~s})$ & $t_{f}(\mathrm{~s})$ & $N_{t}$ & $\xi_{k}=\xi(\%)$ & $\mathrm{N}$ \\
0.95 & 0 & 15 & 10000 & 0.8 & 10 \\
\hline
\end{tabular}

Table 2: Numerical simulation parameters. 


\begin{tabular}{|c|c|c|c|}
\hline \multirow{2}{*}{ Sensor $n^{\circ}$} & \multirow{2}{*}{$\begin{array}{c}\text { Disp. Vel. Acc. } \\
x_{1}(\mathrm{~m})\end{array}$} & \multicolumn{2}{|c|}{ CA-LGFOS } \\
\hline & & $a(\mathrm{~m})$ & $b(\mathrm{~m})$ \\
\hline 1 & 0 & 0 & 0.17 \\
\hline 2 & 0.19 & 0.17 & 0.34 \\
\hline 3 & 0.38 & 0.34 & 0.51 \\
\hline 4 & 0.57 & 0.51 & 0.68 \\
\hline 5 & 0.76 & 0.68 & 0.85 \\
\hline 6 & 0.94 & 0.85 & 1.02 \\
\hline 7 & 1.13 & 1.02 & 1.19 \\
\hline 8 & 1.32 & 1.19 & 1.36 \\
\hline 9 & 1.51 & 1.36 & 1.53 \\
\hline 10 & 1.7 & 1.53 & 1.70 \\
\hline
\end{tabular}

Table 3: CA-LGFOS and standard punctual sensor locations on the clamped-clamped beam. 


\begin{tabular}{|c|c|c|c|c|c|c|c|}
\hline \multirow{2}{*}{ Mode $n^{\circ}$} & \multirow{2}{*}{ Frequency bandwidth $(\mathrm{Hz})$} & \multirow{2}{*}{$N_{c w t}$} & \multirow{2}{*}{$Q$} & \multicolumn{4}{|c|}{$t_{f}(\mathrm{~s})$} \\
\hline & & & & Disp. & Vel. & Acc. & CA-LGFOS \\
\hline 1 & {$\left[\begin{array}{ll}5 & 6\end{array}\right]$} & 200 & 5 & 15 & 13 & 7 & 15 \\
\hline 2 & {$\left[\begin{array}{ll}15 & 16\end{array}\right]$} & 200 & 10 & 5 & 5 & 4 & 5 \\
\hline 3 & {$\left[\begin{array}{ll}29.5 & 30.5\end{array}\right]$} & 200 & 25 & 3 & 3.5 & 4 & 3 \\
\hline
\end{tabular}

Table 4: Parameters of CWT method for local treatment of numerical signals from different measurement types. 


\begin{tabular}{cccccc}
\hline Length & Sheath & Height & Young modulus & Density & Inertia \\
\hline$L(\mathrm{~m})$ & $e(\mathrm{~m})$ & $h(\mathrm{~m})$ & $E_{0}(\mathrm{GPa})$ & $\rho\left(\mathrm{kg} \cdot \mathrm{m}^{-3}\right)$ & $I\left(\mathrm{~m}^{4}\right)$ \\
1 & 0.07 & 0.005 & 2.4 & 1200 & $7.3 \cdot 10^{-10}$ \\
\hline
\end{tabular}

Table 5: Plexiglass cantilever beam characteristics. 


\begin{tabular}{cccccc}
\hline \multirow{2}{*}{ Sensor $n^{\circ}$} & \multicolumn{2}{c}{ Acc. } & \multicolumn{2}{c}{ CA-LGFOS } & \multicolumn{2}{c}{ Strain gauge } \\
\cline { 2 - 6 } & $x_{1}(\mathrm{~m})$ & $a(\mathrm{~m})$ & $b(\mathrm{~m})$ & $a(\mathrm{~m})$ & $b(\mathrm{~m})$ \\
\hline 1 & 0.125 & 0.02 & 0.14 & 0.05 & 0.25 \\
2 & 0.25 & 0.29 & 0.41 & 0.4 & 0.6 \\
3 & 0.375 & 0.56 & 0.68 & 0.76 & 0.96 \\
4 & 0.5 & 0.82 & 0.94 & - & - \\
5 & 0.625 & - & - & - & - \\
6 & 0.75 & - & - & - & - \\
7 & 0.875 & - & - & - & - \\
8 & 1 & - & - & - & - \\
\hline
\end{tabular}

Table 6: CA-LGFOS, accelerometer, and gauge locations on the cantilever beam. 


\begin{tabular}{|c|c|c|c|c|c|c|c|c|c|c|c|c|c|c|c|c|}
\hline \multirow{3}{*}{ Sensor } & \multicolumn{8}{|c|}{ Mode $n^{\circ} 1$} & \multicolumn{8}{|c|}{ Mode $n^{\circ} 2$} \\
\hline & \multicolumn{2}{|c|}{$f_{1}(\mathrm{~Hz})$} & \multicolumn{2}{|c|}{$\Delta \sigma / \sigma(\%)$} & \multicolumn{2}{|c|}{$\xi_{1}(\%)$} & \multicolumn{2}{|c|}{$\Delta \sigma / \sigma(\%)$} & \multicolumn{2}{|c|}{$f_{2}(\mathrm{~Hz})$} & \multicolumn{2}{|c|}{$\Delta \sigma / \sigma(\%)$} & \multicolumn{2}{|c|}{$\xi_{2}(\%)$} & \multicolumn{2}{|c|}{$\Delta \sigma / \sigma(\%)$} \\
\hline & $\mathrm{pp}$ & cwt & $\mathrm{pp}$ & cwt & $\mathrm{pp}$ & cwt & $\mathrm{pp}$ & cwt & $\mathrm{pp}$ & cwt & $\mathrm{pp}$ & cwt & $\mathrm{pp}$ & cwt & $\mathrm{pp}$ & cwt \\
\hline Acc. $n^{\circ} 1$ & 1.15 & 1.20 & 3.3 & 1.6 & 9.0 & 6.4 & 11.4 & 23.5 & 6.17 & 6.15 & 0.1 & 0.1 & 1.3 & 1.1 & 0.6 & 2.1 \\
\hline Acc. $n^{\circ} 2$ & 1.16 & 1.20 & 0.9 & 1.3 & 9.4 & 6.4 & 4.6 & 6.8 & 6.17 & 6.15 & 0.1 & 0.1 & 1.3 & 1.1 & 0.7 & 2.6 \\
\hline Acc. $n^{\circ} 3$ & 1.16 & 1.20 & 1.2 & 1.3 & 9.2 & 6.4 & 3.7 & 6.6 & 6.17 & 6.15 & 0.1 & 0.1 & 1.3 & 1.1 & 0.7 & 2.7 \\
\hline Acc. $n^{\circ} 4$ & 1.16 & 1.20 & 1.2 & 1.3 & 9.2 & 6.4 & 3.9 & 6.5 & 6.17 & 6.15 & 0.1 & 0.1 & 1.3 & 1.1 & 0.7 & 2.6 \\
\hline Acc. $n^{\circ} 5$ & 1.16 & 1.20 & 1.0 & 1.3 & 9.1 & 6.3 & 3.3 & 6.5 & 6.17 & 6.15 & 0.1 & 0.1 & 1.3 & 1.1 & 0.7 & 2.7 \\
\hline Acc. $n^{\circ} 6$ & 1.16 & 1.20 & 1.0 & 1.3 & 9.1 & 6.3 & 3.5 & 6.5 & 6.16 & 6.15 & 0.1 & 0.1 & 1.4 & 1.1 & 1.1 & 2.4 \\
\hline Acc. $n^{\circ} 7$ & 1.16 & 1.20 & 1.0 & 1.3 & 9.1 & 6.3 & 3.4 & 6.4 & 6.18 & 6.15 & 0.1 & 0.1 & 1.2 & 1.1 & 0.5 & 2.8 \\
\hline Acc. $n^{\circ} 8$ & 1.16 & 1.20 & 1.0 & 1.3 & 8.9 & 6.3 & 2.9 & 6.5 & 6.17 & 6.15 & 0.1 & 0.1 & 1.3 & 1.1 & 0.6 & 2.7 \\
\hline average & 1.16 & 1.20 & 1.4 & 1.4 & 9.1 & 6.4 & 4.6 & 8.7 & 6.17 & 6.15 & 0.1 & 0.1 & 1.3 & 1.1 & 0.7 & 2.6 \\
\hline Gauge $n^{\circ} 1$ & 1.13 & 1.20 & 1.1 & 1.6 & 11.3 & 7.3 & 6.9 & 6.2 & 6.17 & 6.15 & 0.1 & 0.1 & 1.3 & 1.1 & 0.7 & 2.6 \\
\hline Gauge $n^{\circ} 2$ & 1.13 & 1.20 & 0.7 & 1.4 & 11.8 & 7.4 & 7.0 & 5.7 & 6.17 & 6.15 & 0.1 & 0.1 & 1.3 & 1.1 & 0.8 & 2.9 \\
\hline Gauge $n^{\circ} 3$ & 1.11 & 1.20 & 0.7 & 1.4 & 12.8 & 7.3 & 7.2 & 5.3 & 6.17 & 6.15 & 0.1 & 0.1 & 1.3 & 1.1 & 0.8 & 2.8 \\
\hline Gauge $n^{\circ} 4$ & 1.09 & 1.20 & 0.8 & 1.3 & 13.3 & 5.9 & 9.5 & 20.9 & 6.17 & 6.15 & 0.1 & 0.1 & 1.4 & 1.1 & 0.8 & 3.1 \\
\hline average & 1.11 & 1.20 & 0.8 & 1.4 & 12.3 & 7.0 & 9.6 & 9.5 & 6.17 & 6.15 & 0.1 & 0.1 & 1.3 & 1.1 & 0.8 & 2.8 \\
\hline FOS $n^{\circ} 1$ & 1.13 & 1.19 & 1.2 & 1.7 & 11.1 & 7.4 & 6.6 & 5.7 & 6.16 & 6.15 & 0.1 & 0.1 & 1.3 & 1.1 & 4.2 & 4.8 \\
\hline FOS $n^{\circ} 2$ & 1.11 & 1.19 & 2.1 & 2.1 & 11.0 & 7.6 & 7.5 & 14.5 & 6.16 & 6.14 & 0.1 & 0.1 & 1.3 & 1.1 & 3.2 & 4.6 \\
\hline FOS $n^{\circ} 3$ & 1.11 & 1.18 & 2.4 & 2.5 & 9.1 & 5.7 & 30.3 & 32.8 & 6.16 & 6.14 & 0.2 & 0.2 & 1.4 & 1.1 & 8.4 & 13.7 \\
\hline average & 1.12 & 1.19 & 1.9 & 2.1 & 10.4 & 6.9 & 14.8 & 17.7 & 6.16 & 6.14 & 0.1 & 0.1 & 1.3 & 1.1 & 5.3 & 7.7 \\
\hline
\end{tabular}

Table 7: Eigenfrequencies and modal damping ratios obtained by peak picking and CWT methods for the first and second modes of the cantilever beam with the accelerometers, strain gauges, and CA-LGFOS. 


\begin{tabular}{|c|c|c|c|c|c|c|c|c|c|c|}
\hline \multirow{3}{*}{ Sensor } & \multicolumn{5}{|c|}{ Mode $n^{\circ} 1$} & \multicolumn{5}{|c|}{ Mode $n^{\circ} 2$} \\
\hline & \multicolumn{2}{|c|}{$\phi_{1_{e x p}}$} & \multicolumn{2}{|c|}{$\Delta \sigma / \sigma(\%)$} & \multirow[t]{2}{*}{$\phi_{1}$} & \multicolumn{2}{|c|}{$\phi_{2 \exp }$} & \multicolumn{2}{|c|}{$\Delta \sigma / \sigma(\%)$} & \multirow[t]{2}{*}{$\phi_{2}$} \\
\hline & pp & cwt & $\mathrm{pp}$ & cwt & & $\mathrm{pp}$ & cwt & $\mathrm{pp}$ & cwt & \\
\hline Acc. $n^{\circ} 1$ & 0.04 & 0.04 & 7.6 & 8.1 & 0.03 & 0.15 & 0.16 & 0.3 & 0.8 & 0.15 \\
\hline Acc. $n^{\circ} 2$ & 0.11 & 0.11 & 0.9 & 0.6 & 0.10 & 0.47 & 0.47 & 0.1 & 0.2 & 0.44 \\
\hline Acc. $n^{\circ} 3$ & 0.22 & 0.22 & 0.7 & 0.4 & 0.20 & 0.73 & 0.74 & 0.1 & 0.2 & 0.70 \\
\hline Acc. $n^{\circ} 4$ & 0.35 & 0.35 & 0.9 & 0.2 & 0.34 & 0.82 & 0.84 & 0.1 & 0.2 & 0.78 \\
\hline Acc. $n^{\circ} 5$ & 0.51 & 0.51 & 0.6 & 0.1 & 0.49 & 0.63 & 0.65 & 0.1 & 0.2 & 0.61 \\
\hline Acc. $n^{\circ} 6$ & 0.66 & 0.67 & 0.8 & 0.1 & 0.66 & 0.21 & 0.22 & 0.2 & 0.6 & 0.20 \\
\hline Acc. $n^{\circ} 7$ & 0.83 & 0.84 & 0.7 & 0.1 & 0.83 & -0.40 & -0.39 & 0.1 & 0.2 & -0.37 \\
\hline \multirow[t]{3}{*}{ Acc. $n^{\circ} 8$} & 1 & 1 & 0 & 0 & 1 & -1 & -1 & 0 & 0 & -1 \\
\hline & \multicolumn{2}{|c|}{$\Phi_{1_{\text {exp }}}$} & \multicolumn{2}{|c|}{$\Delta \sigma / \sigma(\%)$} & $\Phi_{1}$ & \multicolumn{2}{|c|}{$\Phi_{2_{e x p}}$} & \multicolumn{2}{|c|}{$\Delta \sigma / \sigma(\%)$} & $\Phi_{2}$ \\
\hline & $\mathrm{pp}$ & cwt & $\mathrm{pp}$ & cwt & & $\mathrm{pp}$ & cwt & $\mathrm{pp}$ & cwt & \\
\hline Gauge $n^{\circ} 1$ & 1 & 1 & 0 & 0 & 1 & 0.91 & 0.92 & 1.1 & 2.7 & 0.94 \\
\hline Gauge $n^{\circ} 2$ & 0.63 & 0.64 & 0.2 & 0.6 & 0.60 & -0.68 & -0.68 & 0.1 & 0 & -0.67 \\
\hline Gauge $n^{\circ} 3$ & 0.26 & 0.27 & 0.5 & 0.6 & 0.25 & -1 & -1 & 0 & 0 & -1 \\
\hline Gauge $n^{\circ} 4$ & 0.040 & 0.043 & 7.2 & 8.2 & 0.04 & -0.23 & -0.23 & 0.4 & 0.7 & -0.25 \\
\hline FOS $n^{\circ} 1$ & 1 & 1 & 0 & 0 & 1 & 0.41 & 0.41 & 2.7 & 1.4 & 0.46 \\
\hline FOS $n^{\circ} 2$ & 0.42 & 0.43 & 1.7 & 4.2 & 0.44 & -1 & -1 & 0 & 0 & -1 \\
\hline FOS $n^{\circ} 3$ & 0.12 & 0.13 & 5.9 & 23.9 & 0.06 & -0.38 & -0.40 & 2.7 & 6.3 & -0.31 \\
\hline
\end{tabular}

Table 8: Eigenvectors obtained by peak picking and CWT methods for the first and second modes of the cantilever beam with the accelerometers, strain gauges, and CA-LGFOS. 